To be published in Journal of the Optical Society of America B:

Title: $\quad$ Alexandrite: an attractive thin-disk laser material alternative to $\mathrm{Yb}: \mathrm{YAG}$ ?

Authors: $\quad$ Umit Demirbas,Franz Kaertner

Accepted: 17 December 19

Posted 18 December 19

DOI: $\quad$ https://doi.org/10.1364/JOSAB.380140

(C) 2019 Optical Society of America 


\title{
Alexandrite: an attractive thin-disk laser material alternative to Yb:YAG?
}

\author{
UMit DemiRBAS, ${ }^{1,2,{ }^{*}}$ AND Franz X. KÄRTNER ${ }^{1,3}$ \\ ${ }^{1}$ Center for Free-Electron Laser Science, Deutsches Elektronen-Synchrotron DESY, Notkestraße 85, \\ 22607 Hamburg, Germany \\ ${ }^{2}$ Laser Technology Laboratory, Antalya Bilim University, 07190 Dosemealti, Antalya, Turkey \\ ${ }^{3}$ Physics Department and The Hamburg Centre for Ultrafast Imaging, University of Hamburg, Luruper \\ Chaussee 149, 22761 Hamburg, Germany \\ *uemit.demirbas@cfel.de
}

\begin{abstract}
Yb:YAG thin-disk (TD) technology has enabled construction of laser/amplifier systems with unprecedented average/peak power levels, and became the work-horse of many scientific investigations. On the other hand, for some applications, the narrow emission bandwidth of Yb:YAG limits its potential, and search for alternative broadband TD gain media with suitable thermo-opto-mechanical parameters is ongoing. The alexandrite gain medium has a broad emission spectrum centered around $750 \mathrm{~nm}$, possesses thermomechanical strength that even outperforms Yb:YAG, and owns unique spectroscopic properties enabling efficient laser operation even at elevated temperatures. In this work, we have numerically investigated the power scaling potential of continuous-wave (cw) Alexandrite lasers in TD geometry for the first time. Using a detailed laser model, we have compared the potential $\mathrm{cw}$ laser performance of Yb:YAG, Ti:Sapphire, Cr:LiSAF, Cr:LiCAF and Alexandrite thin disk lasers under similar conditions, and show that among the investigated transition metal doped gain media, Alexandrite is the best alternative to $\mathrm{Yb}: \mathrm{YAG}$ in power scaling studies at room temperature. Our analysis further demonstrates that potentially Ti:Sapphire is also a good alternative TD material, but only at cryogenic temperatures. However, in comparison with $\mathrm{Yb}: \mathrm{YAG}$, the achievable laser gain is relatively low for both Alexandrite and Ti:Sapphire, which then requires usage of low-loss cavities with small output coupling, for efficient continuous-wave operation.
\end{abstract}

(c) 2019 Optical Society of America under the terms of the OSA Open Access Publishing Agreement

\section{Introduction}

The thin-disk laser (TDL) concept has strongly boosted the average power levels achievable from solid-state laser oscillators/amplifiers over the last decades [1]. Due to its thermo-optomechanical strength, Yb:YAG has been the workhorse of TD systems [2-4]. Current Yb:YAG TDLs provide $\mathrm{cw}$ power levels up to $10 \mathrm{~kW}$, and $\mathrm{cw}$ mode-locked average power levels around $300 \mathrm{~W}$ ( $275 \mathrm{~W}$ with $583 \mathrm{fs}$ pulses [5], $155 \mathrm{~W}$ with $140 \mathrm{fs}$ pulses [6]). Via regenerative amplification $1.1 \mathrm{ps}$ pulses with $200 \mathrm{~mJ}$ energy at $5 \mathrm{kHz}(1 \mathrm{~kW}$ average power) has also been realized [7]. On the other hand, the narrow emission bandwidth of the Yb:YAG gain medium is an important limitation, restricting obtainable pulse widths and tunability. As a result, research for alternative TDL materials with strong thermo-opto-mechanical parameters and broad emission bandwidth is ongoing.

Part of this search is focused on other $\mathrm{Yb}^{+3}$-doped gain media such as Yb:YLF [8, 9], $\mathrm{Yb}: \mathrm{CALGO}$ [10], and Yb:KYW [11]; however, the emission bandwidth of these materials are also rather limited. As an alternative, transition-metal doped laser materials such as Ti:Sapphire (660-1180 nm) [12-14], Alexandrite (701-858 nm) [15-19], Cr:Forsterite (11301367) [20, 21], Cr:ZnSe (1880-3350 nm) [22, 23], etc... provide significantly broader optical gain bandwidth at different regions of the optical spectrum, and investigation of their TD 
potential is also of great interest [24-26]. In this work, in search for an attractive broadband thin-disk laser material, we have focused our attention to the thermo-mechanically strong Alexandrite $\left(\mathrm{Cr}: \mathrm{BeAl}_{2} \mathrm{O}_{4}\right)$ gain media. Alexandrite was the first lasing material where broadly tunable laser operation could be demonstrated at room temperature (RT), and to our knowledge, it is also the only broadband lasing material where continuous-wave lasing operation could be achieved even with flashlamp pumping [27]. With the progress observed in brightness of laser diodes operating in the red spectral region, Alexandrite has attracted a renewed interest over the last few years [17, 28-38].

In this work, we have numerically investigated the lasing potential of Alexandrite in TD geometry for the first time. In our analysis, to survey Alexandrite's TDL performance in a comparative manner, we have also included Yb:YAG, Ti:Sapphire, Cr:LiCAF and Cr:LiSAF into our investigation. We have started our simulation efforts by examining the roomtemperature $\mathrm{cw}$ laser performance of Yb:YAG and Ti:Sapphire TDLs, where a relatively good-match with earlier experimental results was observed [39-41]. Further analysis clearly showed that, among the broadband laser gain media investigated in this study, Alexandrite is the best candidate for power scaling for room temperature thin disk laser development. Moreover, we have seen that, Ti:Sapphire is also a very good alternative TD material, but only when it is operated at cryogenic temperatures. On the other hand, as a strong shortcoming, we have shown that, the achievable optical gain is rather low in both Alexandrite and Ti:Sapphire, which certainly creates challenges in the design of efficient laser and amplifier systems using these materials.

The paper is organized as follows. In section 2, we discuss pros and cons of Alexandrite in terms of laser related material properties. Then, in Section 3 and 4, we provide details on the investigated TD laser geometry and the numerical model that has been applied. In Section 5, we discuss the advantages of Alexandrite compared to other transition-metal doped gain media by providing continuous-wave lasing simulation results under similar conditions. Lastly, in section 6 , we summarize with a brief conclusion.

\section{Strengths and weaknesses of the Alexandrite gain medium}

In this initial section, before we go into the details of laser performance modeling, we would like to shortly review laser related material properties of Alexandrite to introduce its strengths and weaknesses. Starting from the positive side:

- Alexandrite possesses an intrinsic slope efficiency of 65\% [42], and has broad emission bands enabling tunable lasing in the $700-860 \mathrm{~nm}$ region $[15,16]$. Alexandrite's broad gain spectrum could potentially generate sub-20-fs pulses centered around $750 \mathrm{~nm}$ [43]. Mode-locking of Alexandrite using Saturable Bragg Reflectors (SBRs) [29], Kerr-lensing $[30,31]$, and graphene saturable absorbers [32] has recently been demonstrated, resulting in generation of down to $70-$ fs level pulses so far [31].

- Alexandrite owns a strong and broad absorption band centered around $590 \mathrm{~nm}$, that enables efficient direct diode pumping by red diodes. State-of-the-art pump modules with $100 \mathrm{~W}$ output power around $640 \mathrm{~nm}$ and $681 \mathrm{~nm}$ could readily be used for efficient pumping (the latter wavelength corresponds to in line pumping at the $\mathrm{R}_{1}$ transition [15, 44]). For $681 \mathrm{~nm}$ pumping, the quantum defect is only around $11 \%$, very close to Yb:YAG (9\%), and way better than Ti:Sapphire (35\%).

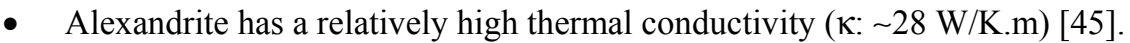

- Alexandrite is mechanically strong (fracture toughness, $\mathrm{K}_{\mathrm{lc}}$, higher than $\mathrm{Yb}: \mathrm{YAG}$ and Ti:Sapphire), and its thermal expansion coefficient $(\alpha)$ and temperature dependent refractive index (dn/dT) values are similar to Yb:YAG [46-48] (see Table 1 for details). Hence, as a first order assessment, thermally induced beam deformations of Alexandrite and $\mathrm{Yb}: Y A G$ TD systems are estimated to be of similar order of magnitude. 
Table 1: Comparison of selected laser related parameters of Alexandrite, Ti:Sapphire and Yb:YAG at room temperature.

\begin{tabular}{|c|c|c|c|}
\hline Gain Medium & Ti:Sapphire & Alexandrite & Yb:YAG \\
\hline Dopant site & $\left(\mathrm{Ti}_{\mathrm{x}} \mathrm{Al}_{1-\mathrm{x}}\right)_{2} \mathrm{O}_{3}$ & $\mathrm{Be}\left(\mathrm{Al}_{1-\mathrm{x}} \mathrm{Cr}_{\mathrm{x}}\right)_{2} \mathrm{O}_{4}$ & $\left(\mathrm{Yb}_{\mathrm{x}} \mathrm{Y}_{1-\mathrm{x}}\right)_{3} \mathrm{Al}_{5} \mathrm{O}_{12}$ \\
\hline $\begin{array}{c}\text { Dopant density at } 1 \% \text { doping } \\
{\left[\times 10^{20} \text { ions } / \mathrm{cm}^{3}\right]}\end{array}$ & $4.7[46]$ & $3.51[49]$ & $1.38[46]$ \\
\hline Birefringence & Negative uniaxial & Biaxial & Isotropic \\
\hline Mass density $\left[\mathrm{g} / \mathrm{cm}^{3}\right], \rho$ & 3.98 & $3.69[50]$ & $4.56[49]$ \\
\hline Melting point $\left[{ }^{\circ} \mathrm{C}\right]$ & 2040 & 1870 & 1970 \\
\hline Specific heat capacity $\left[\mathrm{J} / \mathrm{g}^{\circ} \mathrm{C}\right], \mathrm{Cp}$ & 0.761 & $1.05[47]$ & $0.59[49]$ \\
\hline Knoop hardness $\left[\mathrm{kg} / \mathrm{mm}^{2}\right]$ & $1800(/ / \mathrm{c}), 2200(/ / \mathrm{a})$ & $1600-2300[51]$ & $1320[49]$ \\
\hline Thermal conductivity [W/K.m], $\kappa$ & $\begin{array}{c}30.3(/ / \mathrm{a}), 32.5(/ / \mathrm{c})[52] \\
1000(@ 77 \mathrm{~K})[53]\end{array}$ & $28[45]$ & $10[54]$ \\
\hline Thermal expansion coefficient $\left[\times 10^{-6} / \mathrm{K}\right], \alpha$ & $\begin{array}{c}4.8 \& 5.3[54] \\
0.34(@ 77 \mathrm{~K})[53]\end{array}$ & $\begin{array}{c}5.9(/ / \mathrm{a}), 6.1(/ / \mathrm{b}) \\
6.7(/ / \mathrm{c})[55]\end{array}$ & $6.7[54]$ \\
\hline $\begin{array}{l}\text { Temperature dependence of refractive index } \\
{\left[\times 10^{-6} / \mathrm{K}\right], \mathrm{dn} / \mathrm{dT}}\end{array}$ & $\begin{array}{c}13[46] \\
1.9(@ 77 \mathrm{~K})[53]\end{array}$ & $\begin{array}{c}5.9(/ / \mathrm{a}), 6.9(/ / \mathrm{b}) \\
15.2(/ / \mathrm{c})[55]\end{array}$ & 9.9 [46] \\
\hline Young modulus $\left[\mathrm{x} 10^{9} \mathrm{~Pa}\right], \mathrm{E}$ & 335 & $469[50]$ & 310 [49] \\
\hline Poisson's Ratio, $v$ & 0.29 & 0.3 & $0.3[49]$ \\
\hline Fracture toughness $\left[\mathrm{x} 10^{6} \mathrm{~Pa} \mathrm{~m}^{1 / 2}\right], \mathrm{K}_{1 \mathrm{c}}$ & $2.2[54]$ & $2.6[47]$ & $1.4[54]$ \\
\hline Tensile (fracture) strength $\left[\times 10^{6} \mathrm{~Pa}\right], \sigma_{\mathrm{f}}$ & $440^{\mathrm{a}}$ & $520^{\mathrm{a}}$ & $280^{\mathrm{a}}$ \\
\hline Thermal figure of merit $\left[\mathrm{W} / \mathrm{m}^{1 / 2}\right], \mathrm{R}_{\mathrm{T}}{ }^{\prime}$ & $22[54]$ & $14[47]$ & $5.1[54]$ \\
\hline Thermal shock resistance parameter $[\mathrm{W} / \mathrm{cm}], \mathrm{R}_{\mathrm{T}}$ & $44^{\mathrm{a}}$ & $28^{\mathrm{a}}$ & $10.2^{\mathrm{a}}$ \\
\hline $\begin{array}{l}\text { Maximum temperature difference before } \\
\text { cracking }\left[{ }^{\circ} \mathrm{C}\right], \Delta T_{\text {max,disk }}\end{array}$ & 740 & $\begin{array}{c}460(/ / \mathrm{a} \& / / \mathrm{b}) \\
400(/ / \mathrm{c})\end{array}$ & 380 \\
\hline Quantum Defect (\%), $\mathrm{q}_{\mathrm{d}}$ & 35 & $\begin{array}{l}11(681 \mathrm{~nm}), \\
17(640 \mathrm{~nm})\end{array}$ & 9 \\
\hline Emission cross section $\left[\mathrm{x} 10^{-20} \mathrm{~cm}^{2}\right], \sigma_{\mathrm{em}}$ & $15(/ / a), 41(/ / c)[14]$ & $\begin{array}{c}\sim 0.55 @ 25^{\circ} \mathrm{C} \\
\sim 1.3 @ 100{ }^{\circ} \mathrm{C}(/ / \mathrm{b})\end{array}$ & 2.1 [49] \\
\hline Fluorescence lifetime $[\mu \mathrm{s}], \tau_{\mathrm{f}}$ & $3.2[56]$ & $262[57]$ & $940[46]$ \\
\hline Gain product $\left[\times 10^{-26} \mathrm{~cm}^{2} \mathrm{~s}\right] \sigma_{\mathrm{em}} \tau_{\mathrm{f}}$ & $48(/ / a), 131(/ / \mathrm{c})$ & $\begin{array}{l}130 @ 25^{\circ} \mathrm{C}(/ / \mathrm{b}) \\
163 @ 100^{\circ} \mathrm{C}(/ / \mathrm{b})\end{array}$ & 1975 \\
\hline $\mathrm{T}_{1 / 2}\left[{ }^{\circ} \mathrm{C}\right]$ & $\sim 80[14,39]$ & 530 & - \\
\hline Intrinsic slope efficiency [\%], $\eta_{\mathrm{o}}$ & $64[58]$ & $65[42]$ & $>85[59]$ \\
\hline Relative strength of laser ESA, $\mathrm{f}_{\mathrm{l}}=\sigma_{\mathrm{l}, \mathrm{es} a} \sigma_{\mathrm{em}}$ & $\sim 0[14]$ & $\begin{array}{c}\sim 0.07 @ 25^{\circ} \mathrm{C} \\
\sim 0.17 @ 100{ }^{\circ} \mathrm{C}[60]\end{array}$ & $\sim 0[61]$ \\
\hline Relative strength of pump ESA, $\mathrm{f}_{\mathrm{p}}=\sigma_{\mathrm{p}, \mathrm{esa}} \sigma_{\mathrm{abs}}$ & $\sim 0$ & $\begin{array}{c}\sim 0.7 @ 640 \mathrm{~nm}, \\
\sim 0.01 @ 681 \mathrm{~nm}[62]\end{array}$ & $\sim 0$ \\
\hline Passive losses $[\% / \mathrm{cm}]$ & $2[63]$ & $0.06[42]$ & 0.3 \\
\hline Crystal figure of merit (FOM) & $150[63]$ & $3000[44]$ & 3000 \\
\hline Nonlinear refractive index $\left[\mathrm{x} 10^{-16} \mathrm{~cm}^{2} / \mathrm{W}\right], \mathrm{n}_{2}$ & $3.2[27]$ & $2[50], 3.54[64]$ & $6.9[65]$ \\
\hline Group velocity dispersion $\left(\mathrm{fs}^{2} / \mathrm{mm}\right), \mathrm{GVD}$ & $56.6[66]$ & 60.7 [67] & $66.6[68]$ \\
\hline Third order dispersion $\left(\mathrm{fs}^{3} / \mathrm{mm}\right)$, TOD & $41.4[66]$ & $39.5[67]$ & $66.7[68]$ \\
\hline Gain bandwidth, FWHM (nm) & 260 & 55 & 8 \\
\hline Tuning range $[\mathrm{nm}]$ & $660-1180[12]$ & $\begin{array}{r}701-858 \\
{[15-19]} \\
\end{array}$ & $1016-1108$ \\
\hline Minimum theoretical pulse duration [fs] & 3.5 & 11.9 & 26 \\
\hline Demonstrated shortest pulse duration[fs] & $\sim 5[13]$ & $70[31]$ & $35[69]$ \\
\hline Polarizability difference $\left[\times 10^{-26} \mathrm{~cm}^{3}\right]$ & $\sim-100[70]$ & $\sim 25[71]$ & $1.95[72]$ \\
\hline
\end{tabular}

${ }^{\mathrm{a}}$ We have assumed a maximum flaw size of $50 \mu \mathrm{m}$ in the calculation of tensile strength and thermal shock resistance [26].

- It is possible to grow Alexandrite crystals with very low passive losses, with crystal figure of merit (FOM) values exceeding 3000 (FOM: the ratio of absorption coefficient at the pump wavelength to that at lasing wavelength) [44]. High quality samples with similar FOM are also available in the case of Cr:LiSAF/Cr:LiCAF [25] and Yb:YAG. On the other hand, for Ti:Sapphire due to unavoidable passive losses, the FOM is usually 
limited to around 250, and could at best reach around 500 in hand-selected samples with low doping [63].

- In Alexandrite, nonradiative transitions become effective at very high temperatures and its radiative quantum efficiency is known to be close to unity $(0.95 \pm 0.05)$ for temperatures below $425{ }^{\circ} \mathrm{C}[73,74]$. The critical temperature $\left(\mathrm{T}_{1 / 2}\right)$ at which the nonradiative transitions become as strong as radiative transitions is around $530^{\circ} \mathrm{C}[15$, 74] (compared to 80 in Ti:Sapphire [14, 39]). This enables the escape of the unused inversion as fluorescence, and minimizes the fractional thermal load.

- Alexandrite does not suffer from Auger upconversion process, which is an important limitation in achieving higher laser gain/inversion in Cr:LiCAF and Cr:LiSAF [75].

- For Alexandrite, the maximum temperature difference before cracking (thermomechanical damage) in TD geometry is estimated to be above $400{ }^{\circ} \mathrm{C}[76]$.

- $\mathrm{Cw}$ power levels as high as $26 \mathrm{~W}$, and a slope efficiency of $49 \%$ has already been achieved from a diode-end pumped compact Alexandrite laser in rod geometry [28], showing its great power scaling potential. An average output power of $100 \mathrm{~W}$ was also demonstrated in flashlamp pumped Alexandrite systems $(400 \mathrm{~mJ}$ pulses at $250 \mathrm{~Hz})$, where further power scaling was limited due to thermal distortions, and degradation of output beam quality [77], which could potentially be resolved in TD geometry.

Despite all of the aforementioned advantages, Alexandrite has some important disadvantages as well:

- The gain product of Alexandrite at room-temperature is similar to Ti:Sapphire $\left(\sigma_{\mathrm{em}} \tau_{\mathrm{f}}\right.$ : $130 \times 10^{-26} \mathrm{~cm}^{2} \mathrm{~s}$ ), which is quite low compared to $\mathrm{Yb}: \mathrm{YAG}$. On the other hand, due to the peculiar energy structure of Alexandrite, the $\sigma_{\mathrm{em}} \tau_{\mathrm{f}}$-product increases with temperature, and reaches a value of $163 \times 10^{-26} \mathrm{~cm}^{2} \mathrm{~s}$ at $100{ }^{\circ} \mathrm{C}$ (specified values are for a wavelength of $765 \mathrm{~nm}$ ) [43]. Due to this interesting property, Alexandrite lasers usually provide better laser performance at elevated temperatures ( $\mathrm{cw}$ lasers provide optimum performance around $\left.80-100^{\circ} \mathrm{C}\right)[17,34,35,78]$. The TD laser systems could be designed to operate at temperatures close to $100{ }^{\circ} \mathrm{C}$, to benefit from the increased gain. Note that, the $\sigma_{\mathrm{em}} \tau_{\mathrm{f}}$-product at $100{ }^{\circ} \mathrm{C}$ is still an order of magnitude lower in comparison to $\mathrm{Yb}: \mathrm{YAG}$, which poses a great challenge in TD laser design.

- Alexandrite shows excited state absorption (ESA) both at the pump and lasing wavelength regions $[60,62,79]$. The ratio $\left(f_{p}\right)$ of pump ESA cross section $\left(\sigma_{p, \text { esa }}\right)$ to absorption cross section $\left(\sigma_{\text {abs }}\right)$ is as high as 0.7 at $640 \mathrm{~nm}$. Luckily, at $681 \mathrm{~nm}$, at the inband resonant pumping wavelength, the measured trend in [62] shows that pump ESA gets quite small $\left(f_{p} \sim 0.01-0.015\right)$. The ratio $\left(f_{1}\right)$ of ESA cross section at the lasing wavelength $\left(\sigma_{\mathrm{l}, \mathrm{esa}}\right)$ to emission cross section $\left(\sigma_{\mathrm{em}}\right)$ is relatively low around the gain peak and has a value of 0.05 at room temperature [60]. However, ESA cross section is as strong as the emission cross section at the edges of the emission spectrum, which actually limits the tuning range [43]. Moreover, ESA cross section at the lasing wavelength region is shown to increase with temperature, and the rate of increase in ESA looks higher than the rate of increase observed in emission cross section $[43,79]$.

- For efficient laser operation, the chromium doping of Alexandrite crystals are kept within the $0.01-0.2 \%$ range, due to problems in obtaining uniform doping throughout the laser gain media at higher doping levels. Similarly in Ti:Sapphire, the Ti-doping is kept below $0.25 \%$, since the increased doping levels rises passive losses and reduces the FOM of the crystals [63]. Besides the increased passive losses, high-doping concentration could also produce unfavorable effects such as concentration quenching of fluoresce lifetime, and sharp reduction in thermal merits such as thermal conductivity. Despite these detrimental effects, for Yb:YAG, efficient lasing has been achieved at Yb-doping levels up to 10\% [80]. Interestingly, for Cr:LiCAF and Cr:LiSAF doping up to $100 \%$ is feasible without 
any known significant sacrifice from laser performance at low power levels [81]. Unfortunately, the literature is lacking systematic studies investigating the effect of doping on the laser related parameters for the aforementioned transition-metal doped laser materials, where as more is known for Yb:YAG [82].

- Moreover, in the case of Alexandrite, only around 78\% of the doped $\mathrm{Cr}^{3+}$ ions are on laser active sites [83]. As a result, assuming a reasonable inversion level of $25 \%$, in a $0.2 \% \mathrm{Cr}$-doped Alexandrite crystal, the ion density in the upper laser level is only around $1.37 \times 10^{19}$ ions $/ \mathrm{cm}^{3}\left(0.25 \times 0.2 \times 0.78 \times 3.51 \times 10^{20}\right.$ ions $/ \mathrm{cm}^{3}$ [49]). The emission cross section of Alexandrite at $765 \mathrm{~nm}$ at $100{ }^{\circ} \mathrm{C}$ is around $1.3 \times 10^{-20} \mathrm{~cm}^{2}$, and neglecting ESA, this results in a small signal gain coefficient $\left(\mathrm{g}_{0}\right)$ of only around $0.18 \mathrm{~cm}^{-1}$. For a $100 \mu \mathrm{m}$ thick TD, one bounce form the TD module $(\ell=0,02 \mathrm{~cm})$ will then result in a single pass fractional gain $\left(g_{o} \ell\right)$ of around $0.0036(\sim 0.35 \%)$. Performing a similar analysis for a $10 \%$ Yb-doped $100 \mu \mathrm{m}$ thick Yb:YAG TD, we have found a single pass fractional gain of around $15 \%$. This simple analysis shows the limitation imposed on laser gain by restricted doping capability of Alexandrite compared to $\mathrm{Yb}$ :YAG (assuming similar inversion levels). We also note here that, due to its longer lifetime $(940 \mu \mathrm{s})$, it is easier to achieve high inversion levels in $\mathrm{Yb}: \mathrm{YAG}$ compared to Alexandrite $(260 \mu \mathrm{s})$, providing greater advantage to $\mathrm{Yb}: \mathrm{YAG}$. As an interesting example, for the Ti:Sapphire gain medium, which also could not be highly doped, with the help of the larger emission cross section value, a single pass fractional gain above $25 \%$ is estimated under similar conditions $(0.25 \%$ doped, $100 \mu \mathrm{m}$ thick Ti:Sapphire with an inversion of $25 \%$ ). Unfortunately, as we will see in detail while presenting the laser simulation results, it is not easy (if possible), to reach such inversion levels in roomtemperature operated Ti:Sapphire TD laser systems under cw pumping conditions due its short fluorescence lifetime $(3.2 \mu \mathrm{s})$ and onset of strong nonradiative process upon heating [39].

- For diode pumping of Alexandrite around 640 and $681 \mathrm{~nm}$, only the E//b polarization has significant absorption. Hence, while using un-polarized fiber coupled diode pump modules, combined with limited doping capacity that is discussed above, it is difficult to achieve sufficiently high absorption in TD laser heads.

\section{Details of investigated laser geometry}

In this initial work, we wanted to simplify the laser geometry the TD was explored. The simplest possible geometry with the TD is shown in Fig. 1 (a), where the TD is used as a cavity end mirror. In this configuration, the cavity consists of the gain medium (TD crystal) and an output coupler (both could be either curved or flat) and the number of bounces on the TD in a cavity round trip is one $(\mathrm{m}=1)$. Note, that one passes the gain element twice in every bounce. A slightly more complex cavity form, which is usually employed to improve gain, is as shown in Fig. 1 (b), where the TD is employed as an intracavity mirror; hence, the number of bounces on the TD in every round trip is $2(\mathrm{~m}=2)$. In this form, we assumed that the cavity also contains a curved high reflector. This second system is very similar to what has been used in the initial Ti:Sapphire TDL study [39], and will also be used as a test bed in our current work.

In the cw laser simulations, we have assumed that the cavity facilitate lasing in multimode regime, to enable higher extraction efficiencies [39]. The investigated Alexandrite TD is a ccut sample and the b-axis with the higher emission cross section is employed in lasing. The pump diode provides its output via a multimode fiber, and a multi-pass pump optic module provides up to 72 passes through the TD for efficient (>80\%) absorption of pump light [39, 84]. The pump wavelength is taken as $\sim 681 \mathrm{~nm}$, to enable in-band pumping using the $\mathrm{R}_{1}$ line. For that, the pump diode temperature should be adjustable, so that one can match the diode wavelength to the absorption peak [44]. In most of our analysis, we have adopted a pump 
power level of around $100 \mathrm{~W}$, since to our knowledge red diode modules at similar power levels are already commercially available. On the other hand, it is well-known that, output power levels in TD geometry could be scaled up relatively easily, by employing larger spot sizes on the TD. The pump beam profile on the TD is assumed to be a super Gaussian with order 10. The front and back surfaces of the TD contain the usual antireflective and high reflective coatings for pump and laser wavelengths. All the optics are extremely low-loss to minimize the passive losses $(\mathrm{R}>99.995 \%, \mathrm{AR}<0.005 \%)$, and the maximum flaw size in the TD is assumed to be below $50 \mu \mathrm{m}$. The TD is mounted on a multi-layered $20^{\circ} \mathrm{C}$ water-cooled heat spreader [85] with proper heat extraction capability. For TD laser materials other than Alexandrite, we have assumed usage of similar experimental systems (additional details will be provided while discussing the specific simulation results for each material).
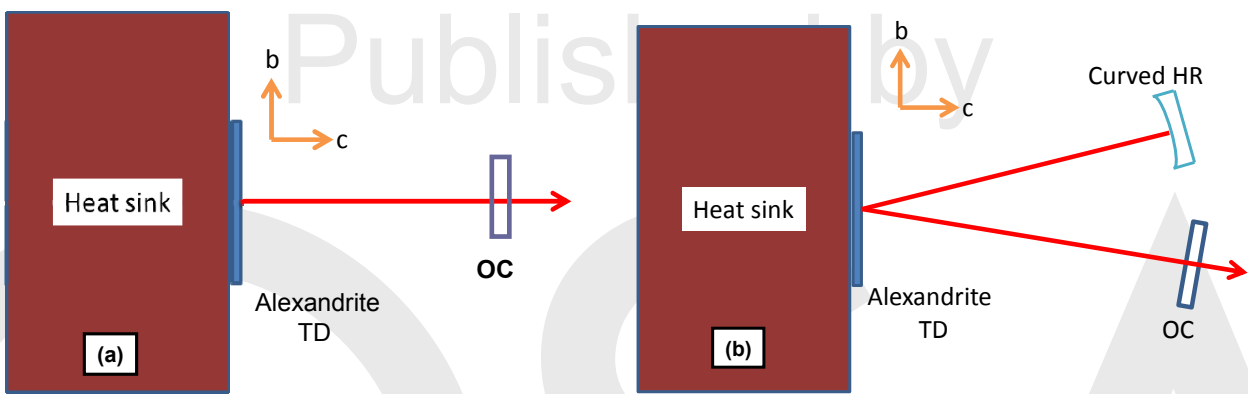

Fig. 1: (a) Schematic of the Alexandrite TDL resonator where the TD acts as an end mirror $(\mathrm{m}=1$ : single bounce on $\mathrm{TD}$ in a round trip). (b) TD is used as an intracavity mirror $(\mathrm{m}=2)$. HR: High reflector. OC: Output coupler.

\section{Details of the numerical model}

We will briefly present the details of the numerical model that was used in estimating the laser performance of Alexandrite TDLs in this section. The numerical method used is similar to what was recently described in [26] for the modeling of Cr:Colquiriite TDLs, but it is an extended and improved version. First of all, for Alexandrite we have to also include the effect of pump excited state absorption and self absorption losses [17, 86, 87]. Additionally, effect of usage of active multipass cell (AMC) cavities with an arbitrary number of bounces in the TD is also included [88-90]. The model that will be outlined below is also valid for the other TD materials that are investigated for the comparative study.

In a TDL cavity, with $m$ bounces on the TD in a cavity round trip, the threshold pump power $\left(\mathrm{P}_{\mathrm{th}}\right)$, the laser slope efficiency $\left(\eta_{\text {eff }}\right)$ and the laser output power $\mathrm{P}_{\text {out }}$ could be estimated using $[86,87,91-93]$ :

$$
\begin{gathered}
P_{t h}=\frac{\pi\left(w_{p}{ }^{2}+w_{c}{ }^{2}\right) h v_{p}}{4 m(M M F)\left(\sigma_{e}-\sigma_{\ell, e s a}+\sigma_{a}\right) \tau_{f}}\left(O C+m L+2 m A_{g}\right) \\
\eta_{e f f}=\left[\left(\frac{h v_{l}}{h v_{p}}\right)\left(\frac{\sigma_{e}-\sigma_{\ell, e s a}}{\sigma_{e}}\right)(M M F)\left(1-\frac{\sigma_{p, e s a}}{\sigma_{e m}} \frac{(O C+m L)}{4 m}\right)\right] \frac{O C}{O C+m L} \\
P_{o u t}=\left(P_{a b s}-P_{t h}\right) \eta_{e f f} .
\end{gathered}
$$

Above $\mathrm{h}$ is Planck's constant, $v_{\mathrm{p}}\left(v_{\mathrm{l}}\right)$ is the pump (laser) photon frequency, $\mathrm{w}_{\mathrm{p}}\left(\mathrm{w}_{\mathrm{c}}\right)$ are the pump (cavity) beam waists, $\sigma_{\mathrm{em}}$ is the effective stimulated emission cross section value at the lasing wavelength $\left(\lambda_{1}\right), \sigma_{1, \text { sesa }}$ is the excited state absorption cross section at $\lambda_{1}, \sigma_{\mathrm{a}}$ is the effective absorption cross section at $\lambda_{1}, \tau_{\mathrm{f}}$ is the effective fluorescence lifetime of the upper laser level, OC is the transmission of the output coupler, $\mathrm{L}$ is the round-trip cavity loss (for 
the simple cavity shown in Fig. 1 (a), with $\mathrm{m}=1$ ), $\mathrm{P}_{\mathrm{abs}}$ is the absorbed pump power, $2 \mathrm{~A}_{\mathrm{g}}$ is the self-absorption losses in the TD at $\lambda_{1}$ in one bounce and MMF is the mode matching factor, that accounts for possible imperfect mode-matching between the laser and pump modes.

In our simulations, MMF factor is taken as $0.75 \mathrm{for} \mathrm{cw}$ operation. The round trip total loss (L) of the simple Alexandrite laser cavity shown in Fig. 1 (a) is assumed to have the following form:

$$
L \cong\left(0.0005+0.05 \mathrm{w}_{p}\right) .
$$

Compared to our earlier work [26], we have chosen to use a more simple loss term in this study to facilitate easier comparison between different TD materials (same loss level is assumed for all the TD materials for simplicity). Note that, in our assumption, the total round trip cavity loss scales linearly with $\mathrm{m}$ due to the increased number of bounces on TD as well as other cavity mirrors (see the factor of $m$ in front of the loss (L) term in Eqs. 1-3). Self absorption loss in one pass through the TD material (Ag) at the lasing wavelength has been estimated using:

$$
A_{g} \approx \sigma_{a} N_{C r} h
$$

where $h$ is the thickness of the $T D$, and $\mathrm{N}_{\mathrm{Cr}}$ is the total laser active ion concentration. The self absorption losses has strong temperature dependence due to the shift of absorption band to longer wavelengths with temperature (or due to the variation of $\sigma_{\mathrm{a}}$ with temperature). The ground state absorption cross section of Alexandrite in the laser wavelength region could be estimated using the extended McCumber theory [94]:

$$
\sigma_{a}(E, T)=\sigma_{e m}(E, T) \operatorname{Exp}\left(\frac{E-E^{*}(T)}{k(T+273)}\right)
$$

where $\mathrm{E}$ is the energy of the photons, $\sigma_{\mathrm{em}}(\mathrm{E})$ is the effective emission cross section value at the energy $\mathrm{E}, \mathrm{k}$ is the Boltzmann constant, $\mathrm{T}$ is the temperature of the crystal in units of ${ }^{\circ} \mathrm{C}$, and $\mathrm{E}^{*}$ is the effective no-phonon energy level. Note that $\mathrm{E}^{*}$ is temperature dependent $[15$, 94] and has a value of $14701 \mathrm{~cm}^{-1}$ at room temperature (corresponds to a wavelength of $680.23 \mathrm{~nm}$ ). The temperature dependence of $\mathrm{E}^{*}$ has been calculated (in $\mathrm{cm}^{-1}$ units) by making a linear fit to the data provided in [79]:

$$
E^{*}(T)=14720.1-0.7 T
$$

The temperature dependence of effective emission cross section of Alexandrite at the lasing wavelength $(\sim 765 \mathrm{~nm})$ has been estimated using [43]:

$$
\sigma_{e m}(T)=0.415+6.28 \times 10^{-3} * T+2.58 \times 10^{-5} * T^{2}+1.16 \times 10^{-8} * T^{3}-6.90 \times 10^{-11} * T^{4} .
$$

We note here that, we have measured the variation of emission cross section between $0-450$ ${ }^{\circ} \mathrm{C}$ ourselves using a method similar to what is employed in [15] to acquire the above formula (this information is also required for Eq. (6)). The temperature dependence of $f_{l}\left(\sigma_{l, \text { esa }} / \sigma_{\text {em }}\right)$ has been estimated using [79]:

$$
\mathrm{f}_{\mathrm{l}}(\mathrm{T})=0.07+0.001 * \mathrm{~T} \text {. }
$$

Unfortunately, high temperature $\left(\mathrm{T}>150{ }^{\circ} \mathrm{C}\right)$ data in [79] has large error bars $( \pm 60 \%)$, but to our knowledge, this is the only data available in the literature. The ratio of $\sigma_{\mathrm{p}, \mathrm{esa}} / \sigma_{\mathrm{em}}$ has been taken as 0.2 and 8 for $681 \mathrm{~nm}$ and $640 \mathrm{~nm}$ pumping respectively (the ratios are assumed to be temperature independent) [62].

In a typical TD, the pump diameter $\left(\mathrm{w}_{\mathrm{p}}\right)$ is much larger than the TD thickness $(\mathrm{h})$, and that results in an almost purely longitudinal heat flow. Under this condition, for a well designed TD cooling geometry with a strong heat extraction capacity (with a large heat transfer coefficient at the boundary), the maximum temperature (T) inside the TD crystal could be roughly estimated using $[85,95,96]$ :

$$
T \cong T_{0}+\frac{1}{2} I_{\text {heat }}\left(R_{\text {disk }}+R_{h s}\right)
$$


where $\mathrm{T}_{0}$ is the cooling water temperature $\left(20{ }^{\circ} \mathrm{C}\right), \mathrm{R}_{\text {disk }}$ is the heat resistance of the disk $(h / \kappa)$, and $\kappa$ is the thermal conductivity of the laser material. Note that, Eq. (10) is estimation for the maximum temperature of the TD, rather than the average value, which we have chosen to use here. This is due to the effectiveness of local heating in diminishing laser performance (as an avalanche mechanism catastrophically reducing performance), especially in systems similar to Cr:LiSAF and Ti:Sapphire with steeply varying fractional thermal load with temperature. For the biaxial Alexandrite gain medium, thermal conductivity in all the

three axis $\left(\kappa_{x},{ }^{\kappa_{y}}\right.$,and $\left.\kappa_{z}\right)$ is reported to be 28 W/K.m [45] at room temperature (hence we can simplify the situation and only use the $\kappa$ term). For a more realistic prediction of the temperatures inside the crystal, we have used an additional heat resistance $\left(\mathrm{R}_{\mathrm{hs}}\right)$ term in $\mathrm{Eq}$ (10), which is due to the heat spreader/sink material and thermal glue/solder used in the TD element (taken as $15 \times 10^{-6} \mathrm{~m}^{2} \mathrm{~K} / \mathrm{W}$ by investigating the data given in Fig. 5 of [39]). The temperature dependence of the thermal conductivity of Alexandrite has been determined using a formula acquired via a making a numerical fit to the data provided in [45]:

$$
\kappa(T)=300000 *(T+273)^{-1.63} \text {, }
$$

where $\mathrm{T}$ is in the units of ${ }^{\circ} \mathrm{C}$, and $\kappa$ is in the units of $\mathrm{W} / \mathrm{Km}$. The heat load per area $\left(\mathrm{I}_{\text {heat }}\right)$ is estimated using:

$$
I_{\text {heat }}=\frac{P_{a b s}}{\pi w_{p}{ }^{2}} \eta_{\text {heat }}
$$

where $\eta_{\text {heat }}$ is the heat generation coefficient, which is calculated with $[97,98]$ :

$$
\eta_{\text {heat }}=1-\frac{\lambda_{p}}{\lambda_{l}}\left(\left(\frac{\sigma_{e}-\sigma_{\ell, e s a}}{\sigma_{e}}\right)\left(1-\frac{\sigma_{p, e s a}}{\sigma_{e m}} \frac{(O C+m L)}{4 m}\right)(M M F) \frac{\frac{\tau_{f}}{\tau_{R}}+\frac{I_{c}}{I_{s a t}}}{1+\frac{I_{c}}{I_{s a t}}}\right) .
$$

In Eq (13), $\mathrm{I}_{\mathrm{c}}$ is the intracavity laser intensity:

and $\mathrm{I}_{\text {sat }}$ is the laser saturation intensity:

$$
I_{c}=2 \frac{P_{o u t}}{\pi w_{c}^{2}} \frac{1}{O C},
$$

$$
I_{s a t}=\frac{h v_{p}}{\left(\sigma_{e m}+\sigma_{a}\right) \tau_{f}} .
$$

The temperature dependence of fluorescence lifetime $\left(\tau_{\mathrm{f}}\right)$ of Alexandrite has been determined with $[43,73,74]$ :

$$
\begin{gathered}
\tau_{\mathrm{F}}=\tau_{\mathrm{R}} \eta_{R E} \\
\tau_{\mathrm{R}}=\tau_{E} \frac{1+\operatorname{Exp}\left(-\frac{\Delta E}{k(T+273)}\right)}{1+\frac{\tau_{E}}{\tau_{T}} \operatorname{Exp}\left(-\frac{\Delta E}{k(T+273)}\right)} \\
\eta_{R E}=\left(1+\frac{\tau_{E}}{\tau_{N R}} \operatorname{Exp}\left(-\frac{\Delta E_{N R}}{k(T+273)}\right)\right),
\end{gathered}
$$

where $\tau_{\mathrm{R}}$ is the effective spontaneous radiative lifetime (which is also temperature dependent in Alexandrite due to the peculiar energy band structure), $\tau_{\mathrm{E}}$ is the lifetime of the storage level $(1.54 \mathrm{~ms}), \tau_{\mathrm{T}}$ is the lifetime of the phonon-broadened upper state laser level $(6.6 \mu \mathrm{s}), \Delta \mathrm{E}$ is the activation energy for phonon coupling between the storage and laser levels $\left(800 \mathrm{~cm}^{-1}\right), \eta_{\mathrm{RE}}$ is the radiative quantum efficiency, $\Delta \mathrm{E}_{\mathrm{NR}}$ is the activation energy for nonradiative process $\left(12000 \mathrm{~cm}^{-1}\right)$, and $\tau_{\mathrm{NR}}$ is the high temperature limit of the nonradiative lifetime $(0.75 \mathrm{ps})$.

In laser efficiency simulations, the coupled set of equations given above are solved iteratively till a stable result is obtained. All the simulations have been performed using 
Mathematica software with a self-written code, which provided us the flexibility to include all the specific fine details of the problem. In the discussion above, we have mostly discussed relevant laser parameters for Alexandrite, which is the focus of this work. Simulation parameters for Yb:YAG, Ti:Sapphire, Cr:LiCAF and Cr:LiSAF is listed in Table 1 and on reference [26], and we also refer the readers to many excellent papers in literature for details $[14,56,99,100]$.

\section{CW lasing simulation results}

In this section, we will be presenting our TD $\mathrm{cw}$ laser performance simulation results in detail. In all of the analysis in this section, we have considered a simple cavity where the TD is acting as an intracavity mirror with 2 bounces on TD in every cavity round-trip (Fig. 1 (b), $\mathrm{m}=2)$, and we have assumed a pump beam diameter of $3 \mathrm{~mm}\left(\mathrm{w}_{\mathrm{p}} \cong \mathrm{w}_{\mathrm{c}}=1.5 \mathrm{~mm}\right)$, a modematching factor (MMF) of 0.75 , a TD thickness of 100 micrometers and a round-trip cavity loss level of around $0.115 \%$ (Eq. 4, taking $\mathrm{w}_{\mathrm{p}}=1.5 \mathrm{~mm}$, and $\mathrm{m}=2$ ). It is obvious that, under real-world conditions, usage of different TD materials results in different operational parameters, but in the analysis of this section, we have assumed similar external parameters for each gain media, and we focus on investigating the effect of intrinsic laser related parameters such as thermal conductivity, emission cross section, and fluorescence lifetime on laser performance. In this way one can clearly link the estimated laser performance to the underlying intrinsic material properties, without the shadowing effect of external parameters. A more detailed study should look at specific specs for each crystal, and could provide optimized results for each material. On the other hand, compared to the main trends that we are aiming to demonstrate here, the additional optimization will only provide incremental improvements, and such a study is beyond the scope of this initial work. Lastly, the cw slope efficiencies that we will present below are with respect to absorbed pump power, and it is assumed that the pump module chamber for each material provides enough passes for efficient absorption of incident pump power.

\subsection{Estimated cw lasing performance of Yb:YAG TD laser}

We start presentation of our simulation results with the well-known Yb:YAG TD laser material[4]. Note that, as required, our model considers the 3-level laser structure of Yb:YAG gain media and accounts for temperature dependence of effective emission cross section, selfabsorption losses and thermal conductivity. Figure 2 shows calculated cw laser slope efficiency curves for $\mathrm{Yb}: \mathrm{YAG}$ (simulation parameters are given in detail in the figure caption, and in Table 1). We see that due to its large gain product, the Yb:YAG laser operates efficiently even with large output coupling. Laser performance with up to $10 \% \mathrm{OC}$ is shown in Fig. 2, but the system enables cw lasing even with output coupling values above $50 \%$ due to the large gain values that could be acquired (as we will see soon this is not the case for the aforementioned transition-metal-doped gain media).

We see from Fig. 2 (b) that, using a 1\% OC, the lasing threshold is estimated to be $15 \mathrm{~W}$, and a slope efficiency as high as $60 \%$ looks feasible. Note that the slope efficiency estimated here is limited mostly by the mode-matching factor $(0.75)$ used in the simulations. Assuming perfect mode-matching $(\mathrm{MMF}=1)$ our model estimates a slope efficiency up to $83 \%$ (this usually comes at the expense of reduced laser output beam quality). Comparing the simulation results with the literature, we see that using a 10\%-doped $110-140 \mu \mathrm{m}$ thick Yb:YAG TD laser material, cw slope efficiencies of $82 \%$ has been achieved from a Yb:YAG laser with multi transverse mode laser output by pumping at the zero-phonon line $(1 \mathrm{~kW}$ output power, $4.5 \%$ output coupler, $1.5 \mathrm{~kW}$ pump power, a pump spot diameter of $5.6 \mathrm{~mm}$ ) [40]. Usually, laser efficiencies decrease to around $50 \%$ level, for cavities that support almost transform limited beam profiles (corresponds to a lower MMF in our simulations) [41]. 

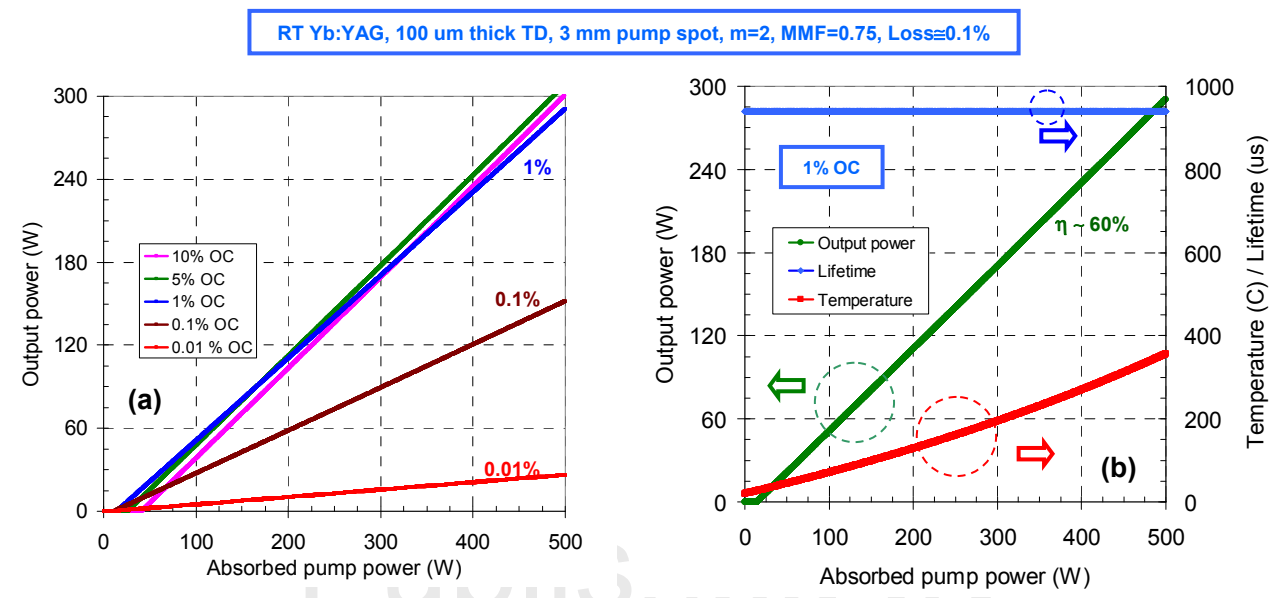

Fig. 2: (a) Calculated cw laser efficiency curves for the Yb:YAG TD laser for output couplers with transmission values in the $0.01 \%$ to $10 \%$ range. The calculations have been performed for a TD cavity with 2 bounces on the TD every round-trip $(\mathrm{m}=2)$, employing a $10 \% \mathrm{Yb}$-doped TD with a thickness of $100 \mu \mathrm{m}$, a pump spot diameter of $3 \mathrm{~mm}$, a mode matching factor (MMF) of 0.75 , and an intracavity loss level of $0.115 \%$. Pump and laser wavelengths are assumed to be $968 \mathrm{~nm}$ and $1030 \mathrm{~nm}$, respectively. (b) Calculated variation of cw laser output power, laser crystal fluorescence lifetime and temperature as a function of absorbed pump power for the Yb:YAG TDL employing the $1 \%$ output coupler. Temperature dependence of thermal conductivity is modeled via $\kappa_{Y b: A G G}(T)=880 *(T+273)^{-0.83}[82,101]$.

Before ending this section, we need to mention that the induced thermal effects might distort the beam quality and lower laser performance at TD temperatures above $\sim 150-200{ }^{\circ} \mathrm{C}$. Hence, the simulation curves in Fig. 2 should be taken as idealistic performance estimates, where the aim is to show any degradation in laser performance due to intrinsic laser related parameters (reduced laser gain with overheating, etc...), and the simulation ignores secondary effects (thermal load causing beam distortions, which then effects laser performance via changing MMF factor, etc..). Note, that we assumed here a fixed fluorescence lifetime of $\mathrm{Yb}$ :YAG independent of temperature (we ignored any nonradiative relaxation processes). There are reports of nonlinear relaxation processes in $\mathrm{Yb}: \mathrm{YAG}$ at high inversion levels [99, 100]. On the other hand, a recent study showed that pumping at the zero-phonon line might suppress these nonradiative processes [101]. Usage of a reasonably-doped TD material and employment of zero-phonon line pumping, mostly validates our assumption here.

\subsection{Estimated cw lasing performance of Ti:Sapphire TD laser}

In this subsection, we will present $\mathrm{cw}$ lasing simulation results of Ti:Sapphire gain medium, under conditions similar to the TD Yb:YAG system that is discussed above. Figure 3 summarizes the expected cw laser performance of Ti:Sapphire TD laser. First of all, due to the $\sim 15$ times smaller gain product compared to $\mathrm{Yb}: \mathrm{YAG}$, the achievable small signal gain is very low in the Ti:Sapphire TD system, and lasing is not possible even with a $1 \%$ output coupler. Moreover, the larger quantum defect (35\% versus $9 \%$ in $\mathrm{Yb}: \mathrm{YAG})$, considerably increases the thermal load on the TD crystal. On top of this, the nonradiative processes are quite effective in Ti:Sapphire even at RT, which considerably limits the achievable inversion (or small signal gain values). All combined this results in limited lasing performance in Ti:Sapphire TD systems that is operated at RT. Note that, the optimum output coupling for the system is only around $0.1 \%$. With this OC, the laser has a lasing threshold of $14 \mathrm{~W}$, and expected slope efficiency is below $20 \%$ (Fig. 3 (b)). Clearly, thermal effects, heating of the crystal and reduction of fluorescence lifetime, considerably limits the laser performance in Ti:Sapphire TDLs operated at RT. Our simulation results matches the recent experimental results obtained with Ti:Sapphire gain medium quite well [39]. With this, we see that, our 
model is effective in successfully estimating the trends of $\mathrm{cw}$ laser performance both for Yb:YAG and Ti:Sapphire TD lasers, despite orders of magnitude difference in performance. We stress here that our aim is to include all the important laser related parameters, but exclude the fine details, and achieve simulation results that could explain the main trends in laser efficiency with each TD material.

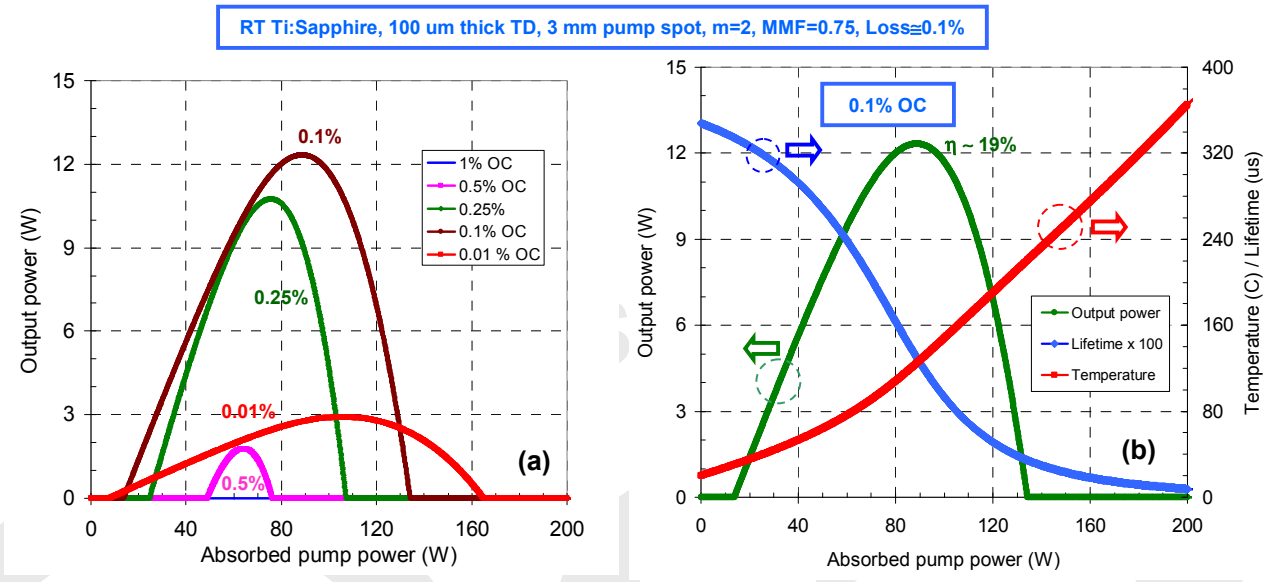

Fig. 3 (a) Calculated cw laser efficiency curves for the RT Ti:Sapphire TD laser for output couplers with transmission values in the $0.01 \%$ to $1 \%$ range. The calculations have been performed for a TD cavity with 2 bounces on the TD every round-trip $(\mathrm{m}=2)$, employing a $0.25 \%$ Ti-doped TD with a thickness of $100 \mu \mathrm{m}$, a pump spot diameter of $3 \mathrm{~mm}$, a mode matching factor (MMF) of 0.75 , and an intracavity loss level of $0.115 \%$. Pump and laser wavelengths are assumed to be $532 \mathrm{~nm}$ and $800 \mathrm{~nm}$, respectively. (b) Calculated variation of cw laser output power, laser crystal fluorescence lifetime and temperature as a function of absorbed pump power for the Ti:Sapphire TD laser employing the $0.1 \%$ output coupler. Note that lifetime $\mathrm{x} 100$ is shown for better visibility in the graph. Temperature dependence of thermal conductivity is modeled assuming $\kappa_{\text {T:Saphire }}(T)=1.26+4070(T+273)^{-1}+1.1 \times 10^{-6}(T+273)^{-2}$.

Cryogenic Ti:Sapphire, 100 um thick TD, $3 \mathrm{~mm}$ pump spot, $m=2, M M F=0.75$, Loss $\cong 0.1 \%$
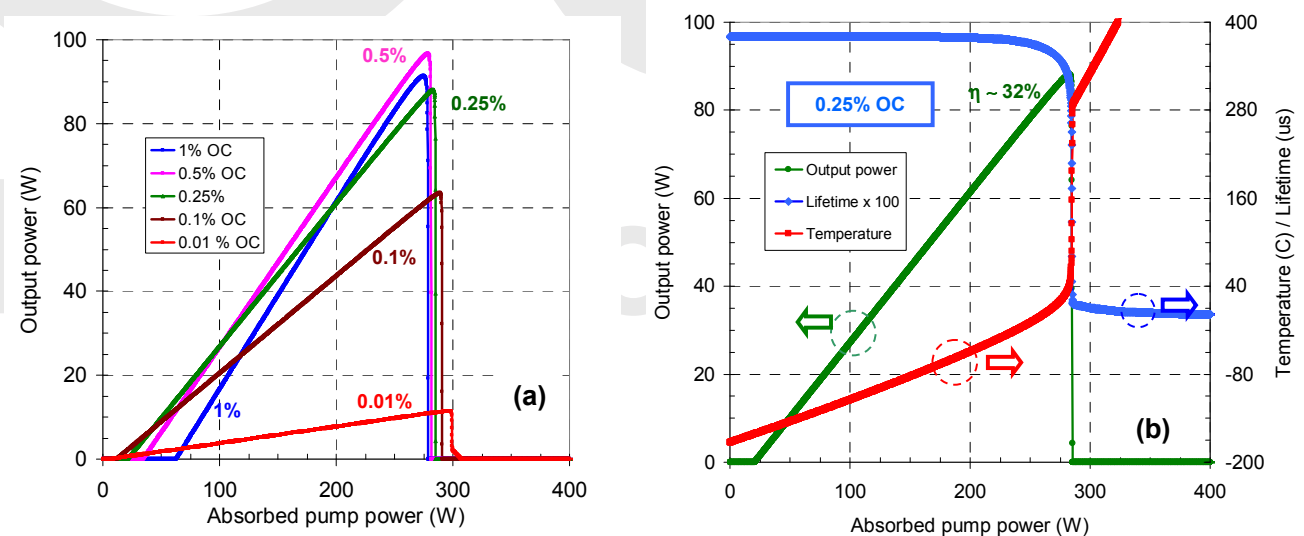

Fig. 4 Calculated cw performance of Ti:Sapphire TD laser when the back side of the heat sink material is cooled to cryogenic temperatures (cooling to $100 \mathrm{~K}$ is assumed here via liquid nitrogen cooling). All the other parameters are same as Fig. 3.

At this point, before moving on with other TD gain media, it is instructive to quickly check the possible performance improvement that could be achieved in TD Ti:Sapphire lasers by cooling it to cryogenic temperatures $(100 \mathrm{~K})$. At cryogenic temperatures, the small signal 
gain [105-107] as well as the thermal conductivity of the Ti:Sapphire gain medium improves $[53,108]$, and as a result thermal distortions are minimized (Table 1). More importantly, the nonradiative transition rates are ignorable at cryogenic temperatures [14]. Benefits of cryogenic cooling in laser [34] and amplifier performance [105, 109-111] has already been demonstrated experimentally in rod geometry ( $54 \mathrm{~W}$ of average power is reported in [111]). Figure 4 summarizes the estimated laser performance in cryogenically cooled Ti:Sapphire TD lasers systems. First of all, compared to RT operation (Fig. 3), due to the increased heat extraction capability, more pump power could be applied to the system. Secondly, the laser efficiency curves get more linear: the nonlinear behavior mostly due to presence of strong thermal quenching of fluorescence lifetime almost disappears. The system also allows usage of higher output coupling (higher inversion levels are viable): lasing is now possible with 3$4 \%$ transmitting output couplers. The simulation results further show that laser power levels above $25 \mathrm{~W}$ could be achieved with slope efficiencies above $30 \%$ in cryogenically cooled Ti:Sapphire laser systems in TD geometry (at $100 \mathrm{~W}$ absorbed pump power level).

\subsection{Estimated cw lasing performance of Cr:LiSAF and Cr:LiCAF TD lasers}

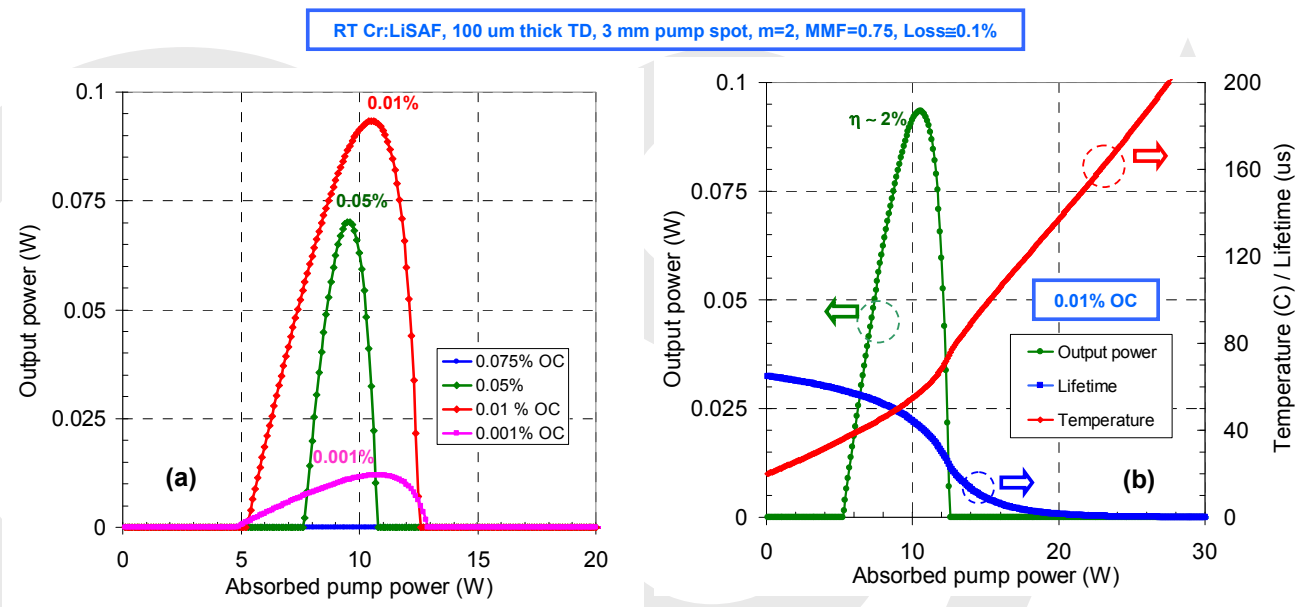

Fig. 5 (a) Calculated cw laser efficiency curves for the Cr:LiSAF TD laser for output couplers with transmission values in the $0.001 \%$ to $0.075 \%$ range. The calculations have been performed for an a-cut, $1 \% \mathrm{Cr}$-doped TD with a thickness of $100 \mu \mathrm{m}$, a pump spot diameter of $3 \mathrm{~mm}$, a mode matching factor (MMF) of 0.75 , and an intracavity loss level of $0.115 \%$. Lasing could not be achieved wit the $0.075 \%$ OC. Pump and laser wavelengths are assumed to be $640 \mathrm{~nm}$ and $850 \mathrm{~nm}$, respectively. (b) Calculated variation of cw laser output power, laser crystal fluorescence lifetime and temperature as a function of pump power for the Cr:LiSAF thin-disk laser for the $0.05 \%$ transmitting output coupler. Temperature dependence of thermal conductivity is modeled assuming $\kappa_{C r-L S A F}(T)=207 *(T+273)^{-0.836}$ [112].

Our detailed TD modeling results with Cr:LiCAF and Cr:LiSAF gain media has been recently presented in [26]; however, the simulation parameters were different than what we use here (slightly lower loss, and slightly higher MMF were considered in [26]), and the simulations will be repeated here to enable a direct comparison with other TD laser materials discussed in this work. We start with Fig. 5, which shows the expected $\mathrm{cw}$ lasing performance of Cr:LiSAF TDL. Due to its low thermal conductivity ( $\kappa: ~ 2.4 \mathrm{~W} / \mathrm{K} . \mathrm{m})$, and susceptibility to temperature quenching of fluorescence lifetime $\left(\mathrm{T}_{1 / 2} \sim 70{ }^{\circ} \mathrm{C}\right)$, the Cr:LiSAF TD heats up very fast before any inversion could be acquired. As a result, the $\mathrm{cw}$ lasing performance is quite poor, and in terms of power scaling, it is the most challenging material among all the gain media investigated in this study [113]. The pump spot size ( $3 \mathrm{~mm}$ diameter) chosen in the comparative simulations is too small for Cr:LiSAF gain media (tight focusing initiates thermal problems). As we have shown in [26], using a much larger spot size (8 $\mathrm{mm}$ diameter), 
output power levels around $10 \mathrm{~W}$ might be feasible in principle (at the expense of further reduction of gain). On the other hand Cr:LiSAF is quite brittle (e.g. fracture toughness 8 times lower than Ti:Sapphire), and handling of such thin and large area TD materials would be very difficult. Lastly, as in the case of Ti:Sapphire, cryogenic operation of Cr:LiSAF might improve its laser performance. However, to our knowledge, the thermo-opto-mechanical parameters of Cr:LiSAF is not yet studied in detail at these temperatures, and hence a reasonable estimate for its laser performance at cryogenic temperatures is not possible at this point.

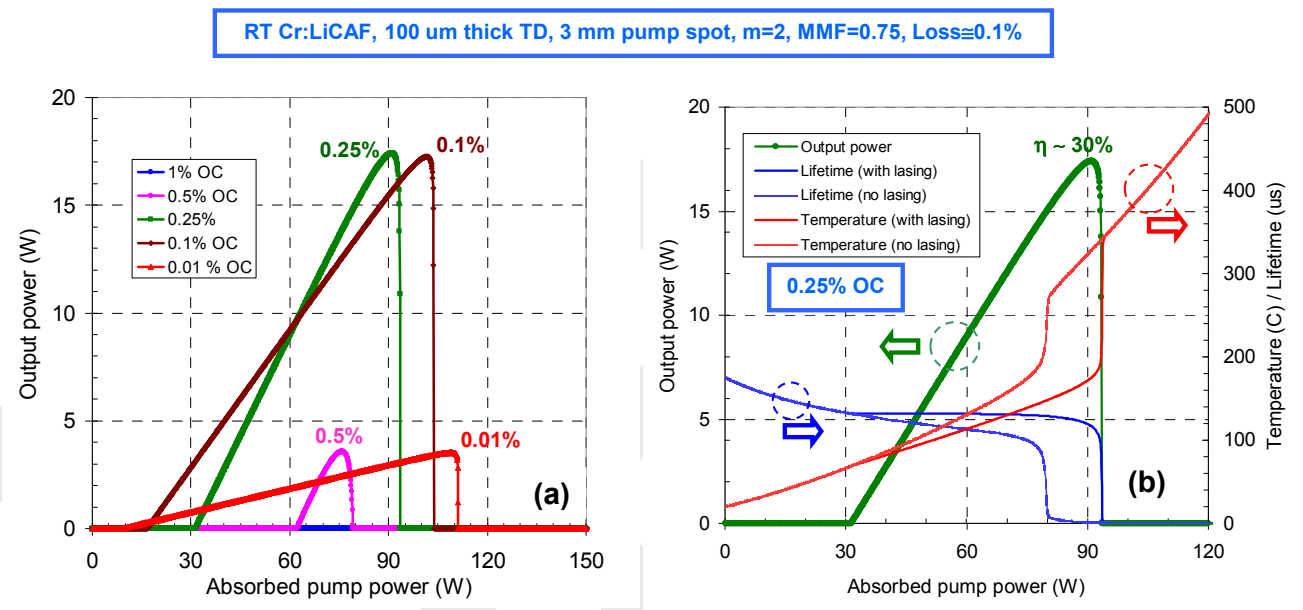

Fig. 6 (a) Calculated cw laser efficiency curves for the Cr:LiCAF TD laser for output couplers with transmission values in the $0.01 \%$ to $1 \%$ range. The calculations have been performed for a c-cut, $2 \% \mathrm{Cr}$-doped TD with a thickness of $100 \mu \mathrm{m}$, a pump spot diameter of $3 \mathrm{~mm}$, a mode matching factor (MMF) of 0.75 , and an intracavity loss level of $0.115 \%$. Lasing could not be achieved wit the $1 \%$ OC. Pump and laser wavelengths are assumed to be 640 $\mathrm{nm}$ and $800 \mathrm{~nm}$, respectively. (b) Calculated variation of $\mathrm{cw}$ laser output power, laser crystal fluorescence lifetime and temperature as a function of pump power for the Cr:LiCAF TD laser for the $0.25 \%$ transmitting OC. Lifetime and temperature has been shown with and without lasing, which shows significant difference due to the presence of Auger upconversion. Temperature dependence of thermal conductivity is modeled via $\kappa_{C r L L C A F}(T)=592 *(T+273)^{-0.836}$.

We continue this subsection with Fig. 6, which shows the expected $\mathrm{cw}$ lasing performance of Cr:LiCAF TD laser. Compared to Cr:LiSAF, Cr:LiCAF has higher thermal conductivity [54, 114], lower quantum defect, and a lower nonradiative decay rate [115], making it a better candidate for power scaling. However, this comes at the expense of a lower gain product, and narrower gain bandwidth. As we can see from Fig. 6, the optimum output coupling of the TD $\mathrm{Cr}$ :LiCAF system is only around $0.25 \%$. With this output coupling, the lasing threshold is estimated to be $32 \mathrm{~W}$, and the slope efficiency of the system reaches to $30 \%$. As discussed in [26], the efficiency of the Cr:LiCAF TD system could be improved by minimizing losses, and improving the mode-matching factor. Further improvement might also be obtained via operating the system also at cryogenic temperatures, but as in the case of Cr:LiSAF, the literature lack data on its properties at cryogenic temperatures, to provide a more in depth analysis here.

\subsection{Estimated cw lasing performance of Alexandrite TD laser}

Figure 7 shows the performance estimate for the cw TD Alexandrite laser. First of all, the achievable small signal gain from the system is relatively high compared to room-temperature Ti:Sapphire, Cr:LiCAF and Cr:LiSAF, but it is still roughly an order of magnitude lower compared to $\mathrm{Yb}: \mathrm{YAG}$. The optimum output coupling is $0.5 \%$, which results in a lasing threshold of around $30 \mathrm{~W}$. A slope efficiency above $40 \%$ also looks feasible form the 
Alexandrite system (for pump power levels up to $100 \mathrm{~W}$, the slope efficiency is $47 \%$, which then reduces to $41 \%$ at high pumping intensities). Note that, as mentioned earlier, in Alexandrite, for temperatures below $400{ }^{\circ} \mathrm{C}$ the decrease in fluorescence lifetime with temperature is not due to the existence of non-radiative processes, but due to the interaction between the ${ }^{2} \mathrm{E}$ and ${ }^{4} \mathrm{~T}_{2}$ levels $[73,74]$. The decrease in lifetime is balanced with an increase in emission cross section rate, and the gain product is maximized at a crystal temperature of around $100{ }^{\circ} \mathrm{C}$. We can see from Fig. 7 (b) that, at a pump power of around $100 \mathrm{~W}$, the Alexandrite crystal temperature is estimated to be around $80{ }^{\circ} \mathrm{C}$, which is close to the optimum temperature for laser performance. As a final note, unlike the other gain media discussed in this work, operating Alexandrite lasers at cryogenic temperatures is not an option for efficiency improvement for the broadband $750 \mathrm{~nm}$ transition. Basically, cooling Alexandrite to cryogenic temperatures minimizes phonon activated coupling of the ${ }^{2} \mathrm{E}$ and ${ }^{4} \mathrm{~T}_{2}$ levels, and prevents accumulation of inversion in the vibronic ${ }^{4} \mathrm{~T}_{2}$ level, that blocks the lasing channel around $750 \mathrm{~nm}$. At cryogenic temperatures, similar to ruby, the Alexandrite medium becomes a 3-level system, and generates narrowband lasing at $680.4 \mathrm{~nm}$ [38].
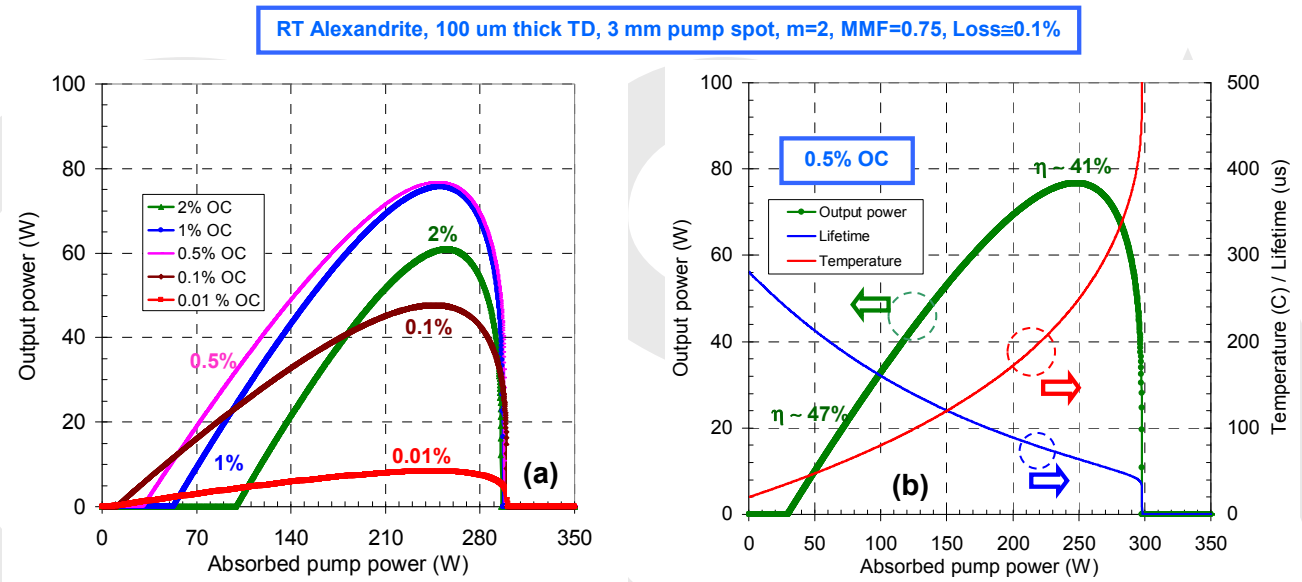

Fig. 7 (a) Calculated cw laser efficiency curves for the Alexandrite TD laser for output couplers with transmission values in the $0.01 \%$ to $1 \%$ range. The calculations have been performed for a c-cut, $0.2 \%$ Cr-doped TD with a thickness of $100 \mu \mathrm{m}$, a pump spot diameter of $3 \mathrm{~mm}$, a mode matching factor (MMF) of 0.75 , and an intracavity loss level of $0.115 \%$. Pump and laser wavelengths are assumed to be $681 \mathrm{~nm}$ and $760 \mathrm{~nm}$, respectively. (b) Calculated variation of $\mathrm{cw}$ laser output power, laser crystal fluorescence lifetime and temperature as a function of pump power for the Alexandrite TD laser for the $0.5 \%$ transmitting output coupler.

\subsection{Comparison of different laser TD gain media under identical conditions}

We are now at a point, where we can look at the estimated TD laser performance of the materials of interest together. Figure 8 shows the expected $\mathrm{cw}$ TD laser performance of Yb:YAG, Alexandrite, Cr:LiCAF, Ti:Sapphire and Cr:LiSAF in the same graph, for a clear direct comparison. In Fig. 8 (a) estimated cw efficiency curves are presented, where as in Fig. 8 (b) and (c) calculated variation of laser crystal temperature and fluorescence lifetime with absorbed pump power levels are shown, respectively. Note that laser performance at the optimum output coupling is shown for each material, where the output coupling is as low as $0.01 \%$ for Cr:LiSAF. Fig. 8 (a) clearly shows the advantages of the Yb:YAG laser system, which outperforms all other candidates in terms of available gain, achievable efficiency and output power levels, but again its narrow gain bandwidth is a key drawback. We see from Fig. 8 that, among the investigated broadband laser materials, with estimated slope efficiencies in the $40-47 \%$ range, Alexandrite is the strongest candidate for power scaling in TD geometry. It is noteworthy to mention that the simulation results of the cryogenically cooled Ti:Sapphire 
TD laser system, with up to around 35\% slope efficiency, is also quite interesting. We can further see from Fig. 8 that, power scaling in TD geometry is quite challenging in room temperature Ti:Sapphire, Cr:LiCAF and especially in Cr:LiSAF. As a side note, in Fig. 8, we have an incident pump power up to $400 \mathrm{~W}$ (in a $3 \mathrm{~mm}$ spot), for all the TD laser materials for comparison purposes. This corresponds to a pump intensity around $5.7 \mathrm{~kW} / \mathrm{cm}^{2}$, which is within the performance limits of Yb:YAG thin disks. However, such pump intensities could not be handled by mechanically soft, thermally unfavorable materials like Cr:LiSAF, Cr:LiCAF, and probably even Ti:Sapphire and Alexandrite might have problems. Hence, especially the temperature calculations in Fig 8 (b) should be considered as curves showing the trend (ignoring TD damage), and as an example, Cr:LiSAF thin disks will probably get damaged at temperatures around $100{ }^{\circ} \mathrm{C}$ already.

100 um thick TD, $3 \mathrm{~mm}$ pump spot, $\mathrm{m}=2$, MMF $=0.75$, Loss $\cong 0.1 \%$
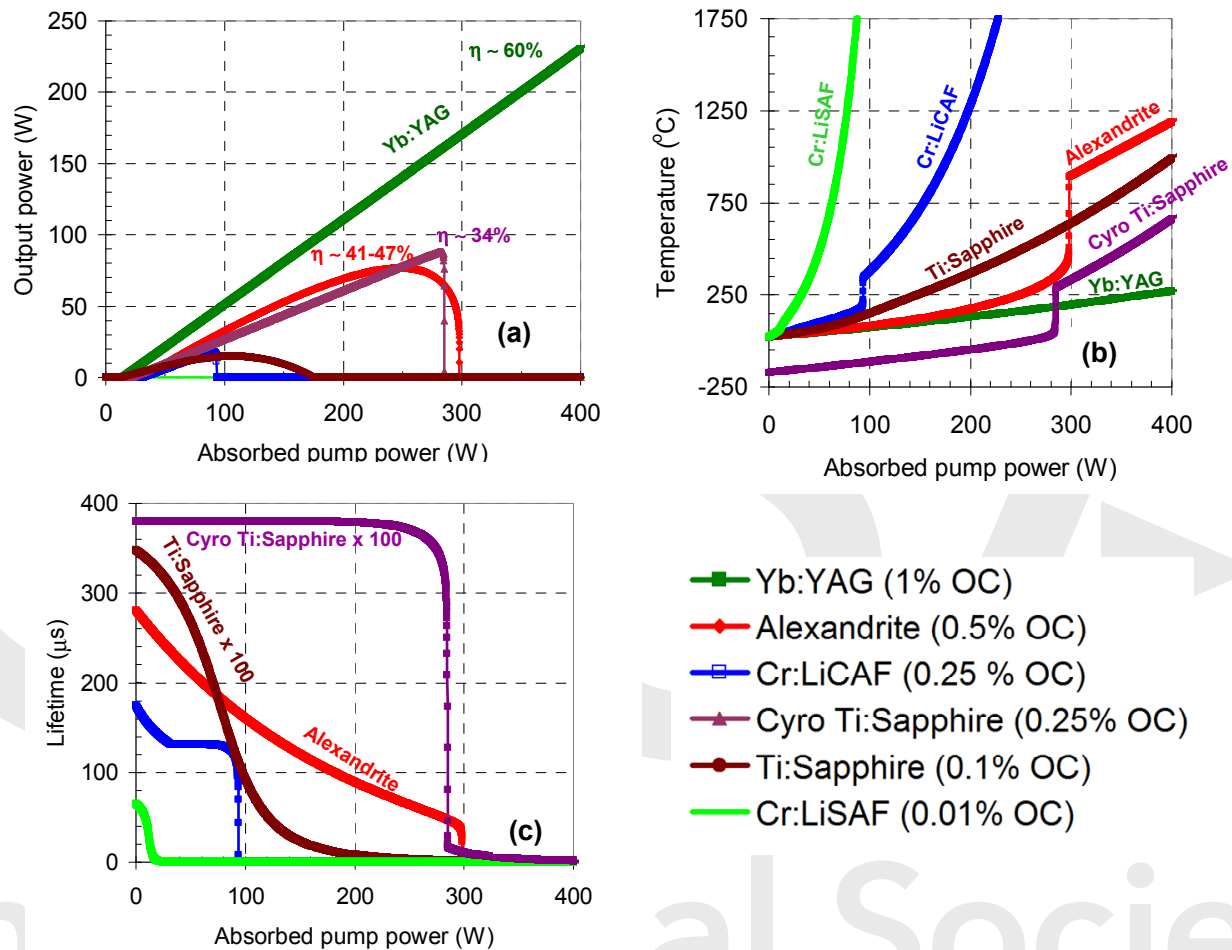

$\rightarrow$ Yb:YAG $(1 \%$ OC)

$\rightarrow$ Alexandrite $(0.5 \% \mathrm{OC})$

$\longrightarrow \operatorname{Cr}: \operatorname{LiCAF}(0.25 \%$ OC)

$\rightarrow$ Cyro Ti:Sapphire $(0.25 \%$ OC)

$\rightarrow$ Ti:Sapphire $(0.1 \%$ OC)

- Cr:LiSAF $(0.01 \%$ OC)

Absorbed pump power (W)

Fig. 8 (a) Calculated cw laser efficiency curves for the Yb:YAG, Alexandrite, Cr:LiCAF, Ti:Sapphire and Cr:LiSAF TD lasers are shown together. The calculations have been performed for a TD thickness of $100 \mu \mathrm{m}$, a pump spot diameter of $3 \mathrm{~mm}$, a mode matching factor (MMF) of 0.75 , and an intracavity loss level of $0.115 \%$. For each material, the data is shown with the optimum output coupling ratio, that is determined from Fig. 2-7. Calculated variation of laser crystal temperature (b) and fluorescence lifetime (c) as a function of absorbed pump power is also shown for the different TD laser materials investigated in this study.

As a summary, in this initial study, we have shown that, with their broad gain bandwidths and favorable thermo-opto-mechanical properties, cryogenically cooled Ti:Sapphire and Alexandrite looks attractive alternatives to Yb:YAG systems. On the other hand, the small signal gain achievable from both of these systems under $\mathrm{cw}$ pumping is rather low compared to $\mathrm{Yb}: \mathrm{YAG}$, and it is clear that special attention will be required in minimizing cavity losses for efficient lasing with Alexandrite and Ti:Sapphire gain media. When we compare RT Alexandrite and cryogenic Ti:Sapphire in terms of cw laser operation, we believe that, with 
the current state-of-the-art technology, Alexandrite TD lasers might be more promising due to: (i) simplicity of room temperature setups compared to cryogenic laser systems, (ii) availability of higher brightness pump diodes, (iii) lower quantum defect (11\% versus $35 \%$ in Ti:Sapphire), and (iv) availability of lower loss crystals. Moreover, our focus in this work is on $\mathrm{cw}$ operation, but amplifier performance of TD systems is also an attractive topic. Unfortunately, due to its short fluoresce lifetime, Ti:Sapphire amplifier systems will require complex pump sources with short intense pulses. In comparison, Alexandrite amplifiers could be pulsed pumped by typical gain-switched laser diode modules, providing it a another advantage compared to Ti:Sapphire.

\section{Conclusions}

In conclusion, to our knowledge, we have presented the first numerical investigation for estimated $\mathrm{cw}$ laser performance of Alexandrite lasers in TD geometry. Comparing Alexandrite with Ti:Sapphire, Cr:LiSAF, Cr:LiCAF, we have shown that, among the gain media investigated, Alexandrite is the best alternative to $\mathrm{Yb}: \mathrm{YAG}$ in power scaling studies at room temperature. We also underline the drawback of Alexandrite in terms of low achievable overall optical gain, which creates challenges in development of efficient laser and amplifier systems.

\section{Funding}

Scientific and Technological Research Council of Turkey (TÜBITAK, 113F199), European Union Marie Curie Career Integration grant (PCIG11-GA-2012-321787), the Alexander von Humboldt Foundation, and Seventh Framework Programme (FP7) FP7/2007- 2013 European Research Council (ERC) (609920).

\section{Acknowledgements}

We would like to thank the three anonymous reviewers whose comments/suggestions helped to significantly improve and clarify this manuscript. UD was supported by the BAGEP Award of the Bilim Akademisi.

\section{Disclosures}

The authors declare no conflicts of interest.

\section{References}

1. A. Giesen, H. Hugel, A. Voss, K. Wittig, U. Brauch, and H. Opower, "Scalable Concept for Diode-Pumped High-Power Solid-State Lasers," Appl. Phys. B 58, 365-372 (1994).

2. C. J. Saraceno, "Mode-locked thin-disk lasers and their potential application for high-power terahertz generation," Journal of Optics 20 (2018).

3. C. J. Saraceno, F. Emaury, C. Schriber, A. Diebold, M. Hoffmann, M. Golling, T. Sudmeyer, and U. Keller, "Toward Millijoule-Level High-Power Ultrafast Thin-Disk Oscillators," IEEE J. Sel. Top. Quantum Electron. 21 (2015).

4. A. Giesen, and J. Speiser, "Fifteen years of work on thin-disk lasers: Results and scaling laws," IEEE J. Sel. Top. Quantum Electron. 13, 598-609 (2007).

5. C. J. Saraceno, F. Emaury, O. H. Heckl, C. R. E. Baer, M. Hoffmann, C. Schriber, M. Golling, T. Sudmeyer, and U. Keller, "275 W average output power from a femtosecond thin disk oscillator operated in a vacuum environment," Opt. Express 20, 23535-23541 (2012).

6. J. Brons, V. Pervak, D. Bauer, D. Sutter, O. Pronin, and F. Krausz, "Powerful 100-fs-scale Kerr-lens modelocked thin-disk oscillator," Opt. Lett. 41, 3567-3570 (2016).

7. T. Nubbemeyer, M. Kaumanns, M. Ueffing, M. Gorjan, A. Alismail, H. Fattahi, J. Brons, O. Pronin, H. G. Barros, Z. Major, T. Metzger, D. Sutter, and F. Krausz, "1 kW, 200 mJ picosecond thin-disk laser system," Opt. Lett. 42, 1381-1384 (2017).

8. H. Cankaya, U. Demirbas, Y. Hua, M. Hemmer, L. E. Zapata, M. Pergament, and F. X. Kärtner, "190-mJ Cryogenically-Cooled Yb:YLF Amplifier System at 1019.7 nm," OSA continuum (2019, in press). 
9. U. Demirbas, H. Cankaya, J. Thesinga, F. X. Kartner, and M. Pergament, "Efficient, diode-pumped, high-power (>300W) cryogenic Yb:YLF laser with broad-tunability $(995-1020.5 \mathrm{~nm})$ : investigation of E//a-axis for lasing " Opt. Express 27, 36562-36579 (2019).

10. S. Ricaud, A. Jaffres, K. Wentsch, A. Suganuma, B. Viana, P. Loiseau, B. Weichelt, M. Abdou-Ahmed, A. Voss, T. Graf, D. Rytz, C. Honninger, E. Mottay, P. Georges, and F. Druon, "Femtosecond Yb:CaGdAlO4 thindisk oscillator," Opt. Lett. 37, 3984-3986 (2012).

11. F. Brunner, T. Sudmeyer, E. Innerhofer, F. Morier-Genoud, R. Paschotta, V. E. Kisel, V. G. Shcherbitsky, N. V. Kuleshov, J. Gao, K. Contag, A. Giesen, and U. Keller, "240-fs pulses with 22-W average power from a mode-locked thin-disk Yb : KY(WO4)(2) laser," Opt. Lett. 27, 1162-1164 (2002).

12. L. G. DeShazer, and K. W. Kangas, "Extended infared operation of titanium sapphire laser," Conference on Lasers and Electro Optics 14, 296-298 (1987).

13. R. Ell, U. Morgner, F. X. Kärtner, J. G. Fujimoto, E. P. Ippen, V. Scheuer, G. Angelow, and T. Tschudi, "Generation of 5 fs pulses and octave-spanning spectra directly from a Ti:sapphire laser," Opt. Lett. 26, 373375 (2001).

14. P. F. Moulton, "Spectroscopic and laser characteristics of Ti: $\mathrm{Al}_{2} \mathrm{O}_{3}$," JOSA B 3, 125-133 (1986).

15. J. C. Walling, O. G. Peterson, H. P. Jenssen, R. C. Morris, and E. W. Odell, "Tunable Alexandrite Lasers," IEEE J. Quantum Electron. 16, 1302-1315 (1980).

16. J. W. Kuper, T. Chin, and H. E. Aschoff, "Extended tuning of Alexandrite laser at elevated temperetures," in Advanced Solid State Lasers(OSA, Salt Lake City, Utah, 1990).

17. W. R. Kerridge-Johns, and M. J. Damzen, "Temperature effects on tunable cw Alexandrite lasers under diode end-pumping," Opt. Express 26, 7771-7785 (2018).

18. I. Yorulmaz, E. Beyatli, A. Kurt, A. Sennaroglu, and U. Demirbas, "Efficient and low-threshold Alexandrite laser pumped by a single-mode diode," Optical Materials Express 4, 776-789 (2014).

19. J. C. Walling, O. G. Peterson, H. P. Jenssen, R. C. Morris, and E. W. O'Dell, "Tunable alexandrite lasers," IEE Journal of Quantum Electronics 16, 1302-1315 (1980).

20. V. G. Baryshevskii, M. V. Korzhik, A. E. Kimaev, M. G. Livshits, V. B. Pavlenko, M. L. Meil'man, and B. I. Minkov, "Tunable chromium forsterite laser in the near IR region," Journal of Applied Spectroscopy (USSR) 53, 675-676 (1990).

21. A. Sennaroglu, Pollock, C. R., Nathel H., "Generation of 48-fs pulses and measurement of crystal dispersion by using a regeneratively initiated self-mode-locked chromium-doped forsterite laser," Opt. Lett. 18, 826-828 (1993).

22. U. Demirbas, and A. Sennaroglu, "Intracavity-pumped $\mathrm{Cr} 2+$ : ZnSe laser with ultrabroad tuning range between 1880 and $3100 \mathrm{~nm}, "$ Opt. Lett. 31, 2293-2295 (2006).

23. E. Sorokin, I. T. Sorokina, M. S. Mirov, V. V. Fedorov, I. S. Moskalev, and S. B. Mirov, "Ultrabroad continuous-wave tuning of ceramic Cr:ZnSe and Cr:ZnS lasers," in OSA / ASSP/LACSEA/LS\&C(OSA, 2010).

24. N. Hodgson, M. Hertwig, and H. Y. Pang, "Transition-metal-doped thin-disk laser," U. S. Patent, ed. (Coherent Inc., USA, 2015).

25. U. Demirbas, "Cr: Colquiriite Lasers: Current Status and Challenges for Further Progress," Progress in Quantum Electronics 68, 100227 (2019).

26. U. Demirbas, "Power scaling potential of continuous-wave Cr:LiSAF and Cr:LiCAF lasers in thin-disk geometry," Appl. Opt. 57, 10207-10217 (2018).

27. E. Sorokin, "Solid-state materials for few-cycle pulse generation and amplification," in Few-cycle laser pulse generation and its applications, F. X. Kärtner, ed. (Springer-Verlag, Berlin, 2004), pp. 3-71.

28. A. Teppitaksak, A. Minassian, G. M. Thomas, and M. J. Damzen, "High efficiency $>26$ W diode end-pumped Alexandrite laser," Opt. Express 22, 16386-16392 (2014).

29. S. Ghanbari, K. A. Fedorova, A. B. Krysa, E. U. Rafailov, and A. Major, "Femtosecond Alexandrite laser passively mode-locked by an InP/InGaP quantum-dot saturable absorber," Opt. Lett. 43, 232-234 (2018).

30. S. Ghanbari, R. Akbari, and A. Major, "Femtosecond Kerr-lens mode-locked Alexandrite laser," Opt. Express 24, 14836-14840 (2016).

31. C. Cihan, A. Muti, I. Baylam, A. Kocabas, U. Demirbas, and A. Sennaroglu, "70 femtosecond Kerr-lens modelocked multipass-cavity Alexandrite laser," Opt. Lett. 43, 1315-1318 (2018).

32. C. Cihan, C. Kocabas, U. Demirbas, and A. Sennaroglu, "Graphene mode-locked femtosecond Alexandrite laser," Opt. Lett. 43, 3969-3972 (2018).

33. U. Parali, X. Sheng, A. Minassian, G. Tawy, J. Sathian, G. M. Thomas, and M. J. Damzen, "Diode-pumped Alexandrite laser with passive SESAM Q-switching and wavelength tunability," Opt. Comm. 410, 970-976 (2018).

34. M. Fibrich, J. Sulc, D. Vyhlidal, H. Jelinkova, and M. Cech, "Alexandrite spectroscopic and laser characteristic investigation within a 78-400 K temperature range," Laser Physics 27 (2017).

35. A. Munk, B. Jungbluth, M. Strotkamp, H. D. Hoffmann, R. Poprawe, J. Hoffner, and F. J. Lubken, "Diodepumped alexandrite ring laser in single-longitudinal mode operation for atmospheric lidar measurements," Opt. Express 26, 14928-14935 (2018).

36. G. Tawy, and M. J. Damzen, "Tunable, dual wavelength and self-Q-switched Alexandrite laser using crystal birefringence control," Opt. Express 27, 17507-17520 (2019).

37. M. Fibrich, J. Šulc, and H. Jelínková, "Alexandrite microchip lasers," Opt. Express 27, 16975-16982 (2019).

38. M. Fibrich, J. Sulc, and H. Jelinkova, "Alexandrite microchip lasers," Opt. Express 27, 16975-16982 (2019). 
39. J. H. Wolter, M. A. Ahmed, and T. Graf, "Thin-disk laser operation of Ti:sapphire," Opt. Lett. 42, 1624-1627 (2017).

40. B. Weichelt, A. Voss, M. A. Ahmed, and T. Graf, "Enhanced performance of thin-disk lasers by pumping into the zero-phonon line," Opt. Lett. 37, 3045-3047 (2012).

41. Y. H. Peng, Y. X. Lim, J. Cheng, Y. Guo, Y. Y. Cheah, and K. S. Lai, "Near fundamental mode $1.1 \mathrm{~kW}$ Yb:YAG thin-disk laser," Opt. Lett. 38, 1709-1711 (2013).

42. R. Scheps, J. F. Myers, T. R. Glesne, and H. B. Serreze, "Monochromatic End-Pumped Operation of an Alexandrite Laser," Opt. Comm. 97, 363-366 (1993).

43. U. Demirbas, A. Sennaroglu, and F. X. Kartner, "Temperature dependence of Alexandrite effective emission cross section and small signal gain over the 25-450 degrees C range," Optical Materials Express 9, 3352-3370 (2019).

44. E. Beyatli, I. Baali, B. Sumpf, G. Erbert, A. Leitenstorfer, A. Sennaroglu, and U. Demirbas, "Tapered diodepumped continuous-wave alexandrite laser," J Opt Soc Am B 30, 3184-3192 (2013).

45. D. A. Vinnik, P. A. Popov, S. A. Archugov, and G. G. Mikhailov, "Heat conductivity of chromium-doped alexandrite single crystals," Doklady Physics 54, 449-450 (2009).

46. T. Sudmeyer, C. Krankel, C. R. E. Baer, O. H. Heckl, C. J. Saraceno, M. Golling, R. Peters, K. Petermann, G. Huber, and U. Keller, "High-power ultrafast thin disk laser oscillators and their potential for sub-100femtosecond pulse generation," Appl. Phys. B 97, 281-295 (2009).

47. B. W. Woods, S. A. Payne, J. E. Marion, R. S. Hughes, and L. E. Davis, "Thermomechanical and thermooptic properties of the $\mathrm{LiCaAlF}_{6}-\mathrm{Cr}^{3+}$ laser material," J Opt Soc Am B 8, 970-977 (1991).

48. P. Loiko, S. Ghanbari, V. Matrosov, K. Yumashev, and A. Major, "Thermo-optical properties of Alexandrite laser crystal," in Solid State Lasers XXVII: Technology and Devices(2018), pp. 105111U-105111-105117.

49. W. Koechner, Solid-state laser engineering (Springer, New York, 2006).

50. J. Yao, and Y. Wang, "Solid Tunable Laser Technology," in Nonlinear Optics and Solid-State Lasers(Springer Series in Optical Sciences, Berlin, Heidelberg, 2012).

51. C. F. Cline, R. C. Morris, M. Dutoit, and P. J. Harget, "Physical-Properties of Beal2o4 Single-Crystals," Journal of Materials Science 14, 941-944 (1979).

52. E. R. Dobrovinskaya, L. A. Lytvynov, and V. Pishchik, Sapphire: Material, Manufacturing, Applications (Springer Science 2009).

53. P. A. Schulz, and S. R. Henion, "Liquid-Nitrogen-Cooled Ti-Al2o3 Laser," IEEE J. Quantum Electron. 27, 1039-1047 (1991).

54. L. J. Atherton, S. A. Payne, and C. D. Brandle, "Oxide and fluoride laser crystals," Annual Review of Materials Science 23, 453-502 (1993).

55. P. Loiko, S. Ghanbari, V. Matrosov, K. Yumashev, and A. Major, "Dispersion and anisotropy of thermo-optical properties of Alexandrite laser crystal," Optical Materials Express 8, 3000-3006 (2018).

56. F. Druon, F. Balembois, and P. Georges, "New laser crystals for the generation of ultrashort pulses," Comptes Rendus Physique 8, 153-164 (2007).

57. J. C. Walling, D. F. Heller, H. Samelson, D. J. Harter, J. A. Pete, and R. C. Morris, "Tunable Alexandrite Lasers - Development and Performance," IEEE J. Quantum Electron. 21, 1568-1581 (1985).

58. A. Sanchez, R. E. Fahey, A. J. Strauss, and R. L. Aggarwal, "Room-temperature continuous-wave operation of a Ti: $\mathrm{Al}_{2} \mathrm{O}_{3}$ laser," Opt. Lett. 11, 363-364 (1986).

59. S. Matsubara, T. Ueda, S. Kawato, and T. Kobayashi, "Highly efficient continuous-wave laser oscillation in microchip Yb : YAG laser at room temperature," Japanese Journal of Applied Physics Part 2-Letters \& Express Letters 46, L132-L134 (2007).

60. M. L. Shand, and J. C. Walling, "Excited-State Absorption in the Lasing Wavelength Region of Alexandrite," IEEE J. Quantum Electron. 18, 1152-1155 (1982).

61. D. C. Brown, R. L. Cone, Y. C. Sun, and R. W. Equall, "Yb : YAG absorption at ambient and cryogenic temperatures," IEEE J. Sel. Top. Quantum Electron. 11, 604-612 (2005).

62. M. L. Shand, J. C. Walling, and R. C. Morris, "Excited-State Absorption in the Pump Region of Alexandrite," J. Appl. Phys. 52, 953-955 (1981).

63. P. W. Roth, A. J. Maclean, D. Burns, and A. J. Kemp, "Directly diode-laser-pumped Ti:sapphire laser," Opt. Lett. 34, 3334-3336 (2009).

64. R. Adair, L. L. Chase, and S. A. Payne, "Nonlinear Refractive-Index of Optical-Crystals," Phys. Rev. B 39, 3337-3350 (1989).

65. C. Honninger, R. Paschotta, M. Graf, F. Morier-Genoud, G. Zhang, M. Moser, S. Biswal, J. Nees, A. Braun, G. A. Mourou, I. Johannsen, A. Giesen, W. Seeber, and U. Keller, "Ultrafast ytterbium-doped bulk lasers and laser amplifiers," Appl. Phys. B 69, 3-17 (1999).

66. X. N. Zhu, J. F. Cormier, and M. Piche, "Study of dispersion compensation in femtosecond lasers," Journal of Modern Optics 43, 1701-1721 (1996).

67. P. Loiko, and A. Major, "Dispersive properties of alexandrite and beryllium hexaaluminate crystals," Optical Materials Express 6, 2177-2183 (2016).

68. D. C. Brown, S. Tornegard, J. Kolis, C. McMillen, C. Moore, L. Sanjeewa, and C. Hancock, "The Application of Cryogenic Laser Physics to the Development of High Average Power Ultra-Short Pulse Lasers," Applied Sciences-Basel 6 (2016). 
69. S. Uemura, and K. Torizuka, "Sub-40-fs Pulses from a Diode-Pumped Kerr-Lens Mode-Locked Yb-Doped Yttrium Aluminum Garnet Laser," Japanese Journal of Applied Physics 50 (2011).

70. R. S. Nagymihaly, H. B. Cao, P. Jojart, V. Zuba, R. Flender, O. Antipov, I. Seres, A. Borzsonyi, V. Chvykov, K. Osvay, and M. Kalashnikov, "Broadband spectral characterization of the phase shift induced by population inversion in Ti:Sapphire," Opt. Express 27, 1226-1235 (2019).

71. V. Pilla, P. R. Impinnisi, and T. Catunda, "Measurement of saturation intensities in ion doped solids by transient nonlinear refraction," Appl. Phys. Lett. 70, 817-819 (1997).

72. I. Tamer, S. Keppler, M. Hornung, J. Korner, J. Hein, and M. C. Kaluza, "Spatio-Temporal Characterization of Pump-Induced Wavefront Aberrations in Yb3+-Doped Materials," Laser \& Photonics Reviews 12 (2018).

73. M. L. Shand, "Quantum Efficiency of Alexandrite," J. Appl. Phys. 54, 2602-2604 (1983).

74. Z. Y. Zhang, K. T. V. Grattan, and A. W. Palmer, "Thermal-Characteristics of Alexandrite Fluorescence Decay at High-Temperatures, Induced by a Visible Laser Diode Emission," J. Appl. Phys. 73, 3493-3498 (1993).

75. U. Demirbas, A. Sennaroglu, F. X. Kartner, and J. G. Fujimoto, "Comparative investigation of diode pumping for continuous-wave and mode-locked Cr3+ : LiCAF lasers," J Opt Soc Am B 26, 64-79 (2009).

76. O. L. Antipov, E. A. Anashkina, and K. A. Fedorova, "Electronic and thermal lensing in diode end-pumped Yb:YAG laser rods and discs," Quantum Electronics 39, 1131-1136 (2009).

77. R. C. Sam, J. J. Yeh, K. R. Leslie, and W. R. Rapoport, "Design and Performance of a $250 \mathrm{~Hz}$ Alexandrite Laser," IEEE J. Quantum Electron. 24, 1151-1166 (1988).

78. S. Guch, and C. E. Jones, "Alexandrite-Laser Performance at High-Temperature," Opt. Lett. 7, 608-610 (1982).

79. M. L. Shand, and H. P. Jenssen, "Temperature-Dependence of the Excited-State Absorption of Alexandrite," IEEE J. Quantum Electron. 19, 480-484 (1983).

80. H. W. Qiu, P. Z. Yang, J. Dong, P. Z. Deng, J. Xu, and W. Chen, "The influence of Yb concentration on laser crystal Yb : YAG," Materials Letters 55, 1-7 (2002).

81. L. K. Smith, S. A. Payne, W. F. Krupke, L. D. DeLoach, R. Morris, E. W. O'Dell, and D. J. Nelson, "Laser emission from the transition-metal compound LiSrCrF6," Opt. Lett. 18, 200-202 (1993).

82. R. L. Aggarwal, D. J. Ripin, J. R. Ochoa, and T. Y. Fan, "Measurement of thermo-optic properties of Y3A15O12, Lu3A15O12, YAIO(3), LiYF4, LiLuF4, BaY2F8, KGd(WO4)(2), and KY(WO4)(2) laser crystals in the 80-300 K temperature range," J. Appl. Phys. 98 (2005).

83. R. C. Powell, L. Xi, X. Gang, G. J. Quarles, and J. C. Walling, "Spectroscopic Properties of Alexandrite Crystals," Phys. Rev. B 32, 2788-2797 (1985).

84. J. W. Zhang, F. Schulze, K. F. Mak, V. Pervak, D. Bauer, D. Sutter, and O. Pronin, "High-Power, HighEfficiency Tm:YAG and Ho:YAG Thin-Disk Lasers," Laser \& Photonics Reviews 12 (2018).

85. N. Hodgson, and A. Caprara, "Semi-analytical solution for the temperature profiles in solid-state laser disks mounted on heat spreaders," Appl. Opt. 55, 5110-5117 (2016).

86. W. R. Kerridge-Johns, and M. J. Damzen, "Analytical model of tunable Alexandrite lasing under diode endpumping with experimental comparison," J Opt Soc Am B 33, 2525-2534 (2016).

87. W. R. Kerridge-Johns, and M. J. Damzen, "Analysis of pump excited state absorption and its impact on laser efficiency," Laser Physics Letters 12 (2015).

88. D. Bauer, I. Zawischa, D. H. Sutter, A. Killi, and T. Dekorsy, "Mode-locked Yb:YAG thin-disk oscillator with $41 \mathrm{mu}$ J pulse energy at $145 \mathrm{~W}$ average infrared power and high power frequency conversion," Opt. Express 20 (2012).

89. J. Neuhaus, J. Kleinbauer, A. Killi, S. Weiler, D. Sutter, and T. Dekorsy, "Passively mode-locked Yb : YAG thin-disk laser with pulse energies exceeding $13 \mathrm{mu} \mathrm{J}$ by use of an active multipass geometry," Opt. Lett. 33, 726-728 (2008).

90. J. Neuhaus, D. Bauer, J. Kleinbauer, A. Killi, D. H. Sutter, and T. Dekorsy, "Numerical analysis of a subpicosecond thin-disk laser oscillator with active multipass geometry showing a variation of pulse duration within one round trip," J Opt Soc Am B 27, 65-71 (2010).

91. D. Findlay, and R. A. Clay, "The measurement of internal losses in 4-level lasers," Physics Letters 20, 277-278 (1966).

92. J. A. Caird, L. G. DeShazer, and J. Nella, "Characteristics of room-temperature $2.3-\mu \mathrm{m}$ laser emission from $\mathrm{Tm}^{3+}$ in YAG and $\mathrm{YAlO}_{3}$," IEEE J. Quantum Electron. 11, 874-881 (1975).

93. A. Sennaroglu, "Classification of power-degrading mechanisms in an optically pumped four-level laser: an analytical approach," J Opt Soc Am B 36, 2202-2209 (2019).

94. M. L. Shand, J. C. Walling, and H. P. Jenssen, "Ground-State Absorption in the Lasing Wavelength Region of Alexandrite - Theory and Experiment," IEEE J. Quantum Electron. 18, 167-169 (1982).

95. V. Ashoori, M. Shayganmanesh, and S. Radmard, "Heat Generation and Removal in Solid State Lasers," in $A n$ Overview of Heat Transfer Phenomena, S. N. Kazi, ed. (IntechOpen, 2012).

96. N. Hodgson, and A. Caprara, "Calculation of temperature profiles in solid-state laser disks mounted on multilayered heat spreaders using Hankel transforms," Appl. Opt. 55, 10146-10157 (2016).

97. A. Sennaroglu, "Broadly tunable $\mathrm{Cr}^{4+}$-doped solid-state lasers in the near infrared and visible," Progress in Quantum Electronics 26, 287-352 (2002).

98. M. Okida, M. Itoh, T. Yatagai, H. Ogilvy, J. Piper, and T. Omatsu, "Heat generation in Nd doped vanadate crystals with 1.34 mu m laser action," Opt. Express 13, 4909-4915 (2005).

99. K. Contag, M. Karszewski, C. Stewen, A. Giesen, and H. Hugel, "Theoretical modelling and experimental investigations of the diode-pumped thin-disk Yb : YAG laser," Quantum Electronics 29, 697-703 (1999). 
100. M. Wang, G. Z. Zhu, X. Zhu, Y. Q. Chen, J. Dong, H. L. Wang, and Y. F. Qian, "Thickness optimization for an anti-ASE cap in a thin disk laser considering dioptric power and aberration-induced loss," J Opt Soc Am B 35, 583-592 (2018).

101. A. K. Jafari, and M. Aas, "Continuous-wave theory of Yb:YAG end-pumped thin-disk lasers," Appl. Opt. 48, 106-113 (2009).

102. I. I. Kuznetsov, I. B. Mukhin, D. E. Silin, A. G. Vyatkin, O. L. Vadimova, and O. V. Palashov, "Thermal Effects in End-Pumped Yb:YAG Thin-Disk and Yb:YAG/YAG Composite Active Element," IEEE J. Quantum Electron. 50, 133-140 (2014).

103. A. Pirri, G. Toci, D. Alderighi, and M. Vannini, "Effects of the excitation density on the laser output of two differently doped Yb:YAG ceramics," Opt. Express 18, 17262-17272 (2010).

104. M. Smrz, T. Miura, M. Chyla, S. Nagisetty, O. Novak, A. Endo, and T. Mocek, "Suppression of nonlinear phonon relaxation in Yb:YAG thin disk via zero phonon line pumping," Opt. Lett. 39, 4919-4922 (2014).

105. A. Dantan, J. Laurat, A. Ourjoumtsev, R. Tualle-Brouri, and P. Grangier, "Femtosecond Ti : Sapphire cryogenic amplifier with high gain and MHz repetition rate," Opt. Express 15, 8864-8870 (2007).

106. H. Burton, C. Debardelaben, W. Amir, and T. A. Planchon, "Temperature dependence of Ti:Sapphire fluorescence spectra for the design of cryogenic cooled Ti:Sapphire CPA laser," Opt. Express 25, 6954-6962 (2017).

107. S. Backus, M. Kirchner, R. Lemons, D. Schmidt, C. Durfee, M. Murnane, and H. Kapteyn, "Direct diode pumped Ti:sapphire ultrafast regenerative amplifier system," Opt. Express 25, 3666-3674 (2017).

108. J. Hidde, C. Guguschev, S. Ganschow, and D. Klimm, "Thermal conductivity of rare-earth scandates in comparison to other oxidic substrate crystals," Journal of Alloys and Compounds 738, 415-421 (2018).

109. I. Matsushima, H. Yashiro, and T. Tomie, "10 kHz $40 \mathrm{~W}$ Ti : sapphire regenerative ring amplifier," Opt. Lett. 31, 2066-2068 (2006).

110. H. Kiriyama, M. Mori, Y. Nakai, T. Shimomura, M. Tanoue, A. Akutsu, H. Okada, T. Motomura, S. Kondo, S. Kanazawa, A. Sagisaka, J. Ma, I. Daito, H. Kotaki, H. Daido, S. Bulanov, T. Kimura, and T. Tajima, "Generation of high-contrast and high-intensity laser pulses using an OPCPA preamplifier in a double CPA, Ti:sapphire laser system," Opt. Comm. 282, 625-628 (2009).

111. I. Matsushima, H. Yashiro, and T. Tomie, "10 kHz $54 \mathrm{~W}$ Ti:sapphire regenerative amplifier as a pumping laser of a laser-plasma x-ray source," in Advanced Laser Technologies(SPIE, 2007), p. 70220M.

112. V. Pilla, T. Catunda, S. M. Lima, A. N. Medina, M. L. Baesso, H. P. Jenssen, and A. Cassanho, "Thermal quenching of the fluorescence quantum efficiency in colquiriite crystals measured by thermal lens spectrometry," J Opt Soc Am B 21, 1784-1791 (2004).

113. U. Demirbas, and I. Baali, "Power and efficiency scaling of diode pumped Cr: LiSAF lasers: $770-1110 \mathrm{~nm}$ tuning range and frequency doubling to 387-463 nm," Opt. Lett. 40, 4615-4618 (2015).

114. A. Steimacher, O. A. Sakai, A. C. Bento, M. L. Baesso, A. N. Medina, S. M. Lima, and T. Catunda, "Angular dependence of the thermal-lens effect on $\operatorname{LiSrAlF}(6)$ and $\operatorname{LiSrGaF}(6)$ single crystals," Opt. Lett. 33, 1720-1722 (2008).

115. M. Stalder, M. Bass, and B. H. T. Chai, "Thermal quenching of fluoresence in chromium-doped fluoride laser crystals," J. Opt. Soc. Am. B 9, 2271-2273 (1992).
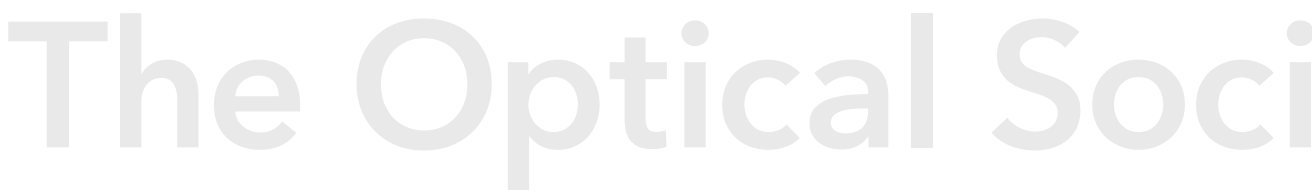